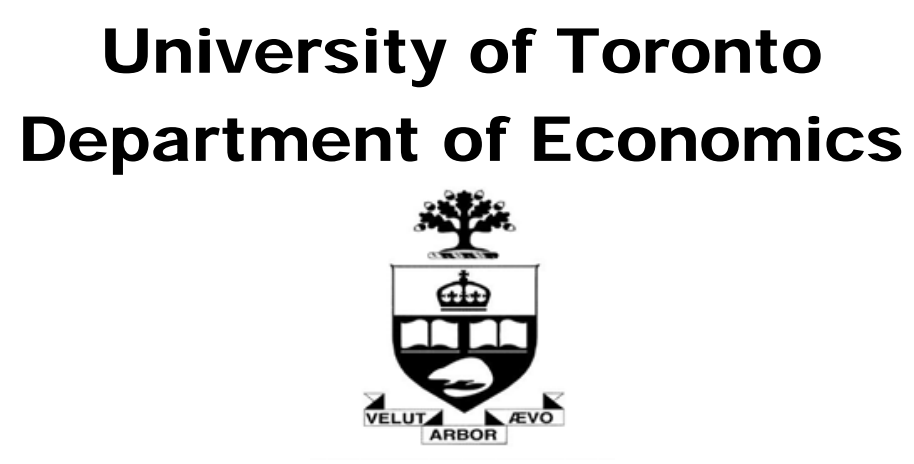

Working Paper 606

Unobserved Heterogeneity in Auctions under Restricted Stochastic Dominance

\author{
By Yao Luo
}

May 19, 2018 


\title{
Unobserved Heterogeneity in Auctions under Restricted Stochastic Dominance
}

\author{
Yao Luo* \\ University of Toronto
}

April 25, 2017

\begin{abstract}
We study the identification of first-price auctions with nonseparable unobserved heterogeneity. In particular, we extend $\mathrm{Hu}$, McAdams, and Shum (2013) by relaxing the first-order stochastic dominance condition. Instead, we assume restricted stochastic dominance relations among the value quantile functions and show that the same relations pass to the bid quantile functions. An ordered tree summarizes these relations and provides a total ordering. Relying on the proposed restricted stochastic dominance ordering, we extend a list of identification results in the empirical auction literature.
\end{abstract}

Keywords: Restricted Stochastic Dominance, Unobserved Heterogeneity, Identification, Misclassification, Auction, Risk Aversion

JEL: C14, D44

\footnotetext{
*Email: yao.luo@utoronto.ca

I would like to thank the editor and anonymous referees for comments that have greatly improved the article. I am indebted to Isabelle Perrigne and Quang Vuong for their advice and encouragement. I also thank Victor Aguirregabiria, Yonghong An, Gaurab Aryal, Xiaohong Chen, Serafin Grundl, Jiaying Gu, Emmanuel Guerre, Marc Henry, Nail Kashaev, Joris Pinkse, Matthew Shum, Yuanyuan Wan, Ruli Xiao, Yu Zhu and conference participants at the 2016 Shanghai Econometrics Workshop and the 2016 CESG for helpful comments. Jan Victor Dee provided excellent research assistance. I thank SSHRC grant 430-2016-00077 for financial support. All errors are my own.
} 


\section{Introduction}

In this paper, we focus on the identification of first-price auctions with unobserved heterogeneity, ${ }^{1}$ which is prominent in many applications. There are two related methods for dealing with unobserved heterogeneity in auctions. The first one is the deconvolution approach. See Li and Vuong (1998), Li, Perrigne, and Vuong (2000) and Krasnokutskaya (2011), among others. They require two bids in each auction and assume that unobserved heterogeneity has a separable effect on bidders' values. The second one is the misclassification approach of $\mathrm{Hu}$, McAdams, and Shum (2013). They consider discrete one-dimensional unobserved heterogeneity and achieve identification with at least three bidders per auction relying on the results of $\mathrm{Hu}(2008)$.

We generalize $\mathrm{Hu}$, McAdams, and Shum (2013) by relaxing the first-order stochastic dominance ordering on the value distributions that they assume. While this ordering arises naturally (e.g., Aradillas-López, Gandhi, and Quint (2013)) and has been verified in some applications (e.g., An, Hu, and Shum (2010)), our relaxation further expands the breadth of applicability of their misclassification approach. In particular, we show that their approach applies as long as a regular full-rank condition and a restricted stochastic dominance (hereafter RSD) ordering on the value distributions are satisfied. The RSD ordering is more general than the first-order stochastic dominance ordering, and it allows all analytic functions as well as some non-analytic functions.

One way to understand our RSD ordering is through introducing an ordered tree for a set of distinct functions. This ordered tree simply describes how each function distinguishes itself from the rest. It contains the points where two or more functions split and their ranking in the neighborhood of these splitting points. This concept allows a novel way of ordering functions. First, it is well-defined for any set of distinct functions under the RSD condition. An ordered tree describes an ordering of the original functions, even in the absence of stochastic dominance of any order. In other words, our ordering of functions relies on a

\footnotetext{
${ }^{1}$ We will use unobserved heterogeneity and state interchangeably.
} 
known functional that yields the ordering when applied to the set of value functions. On the other hand, Hu, McAdams, and Shum (2013) rely on a monotonicity condition to order the value distributions conditional on the state. This requires a known functional that is applied to each value function and the resulting values are increasing in the state. Depending on the kind of unobserved heterogeneity, the exact functional has to be determined case by case.

The advantage of the RSD ordering is threefold. First, it is easy to interpret. Similar to the first-order stochastic dominance ordering, the RSD ordering is equivalent to expected utility ordering (in a limited range) with an increasing utility function. Second, it allows unambiguous ranking of distribution functions in a wider range of models. The first-order stochastic dominance ordering focus on one-dimensional unobserved heterogeneity. However, many commonly used distributions, such as the beta distribution, the two-sided power distribution and the normal distribution, have two or more parameters. Third, it leads to more convenient comparative statics in auctions. The misclassification approach identifies auctions with unobserved heterogeneity in two steps: first identify the component bid distributions and then identify the model primitives. Comparative statics are essential to link the component bid distributions to the model primitives. Since the RSD ordering only requires comparing functions in a limited range, comparative statics are clearer under normally much weaker conditions. On the other hand, the first order stochastic dominance ordering either excludes the model or requires more assumptions on the primitives. To fix ideas, we demonstrate several applications.

First, we prove identification of first-price auction models with unobserved heterogeneity when values are i.i.d. draws from Beta distributions. Note that this family of distributions cannot be ranked by the first-order stochastic dominance relations. However, we find that our RSD ordering is equivalent to a lexicographical ordering of the two shape parameters of Beta distributions. This leads to full identification of models with Beta distributed values, rather than identification up to permutation. Moreover, we obtain a new sufficient condition for nonparametric identification of the same model with covariates. 
Second, we extend the identification results in Guerre, Perrigne, and Vuong (2009) by introducing nonseparable unobserved heterogeneity as in Hu, McAdams, and Shum (2013). We show a novel comparative statics that the same relation of RSD passes from the value quantile functions to the bid quantile functions in first-price auctions. Therefore, the RSD ordering and the ordered tree are both preserved by the mapping from value quantile function to bid quantile function. Moreover, this property is invariant to the number of bidders as well as their utility function. We rely on this result to match the two bid distributions resulting from the same value distribution but different numbers of bidders. This allows us to identify the model primitives relying on Guerre, Perrigne, and Vuong (2009).

Third, we show that the identification results of Gentry and Li (2014) extend to unobserved heterogeneity under a smaller set of assumptions than what they make. They study identification of the Affiliated-Signal model under risk neutrality. Point identification relies on a continuous cost shifter, which reduces to a problem of finite mixture with covariates and suffers from a "label switching" problem. Gentry and Li (2014) make two monotonicity assumptions on the value distributions and entry thresholds to ensure first-order stochastic dominance ordering of the component bid distributions. Relying on the proposed RSD ordering, we show that their identification results still hold without the assumption on the ordering of value distributions.

Fourth, we consider an example of multidimensional unobserved heterogeneity. ${ }^{2}$ Unobserved heterogeneity arises for a wide range of reasons. Examples include unknown number of bidders (An, Hu, and Shum (2010)), implicit reserve price or bidding cost (Hu, McAdams, and Shum (2013)), unknown bidders' types or bounded rationality (An (2016)) and multiple equilibria (Xiao (2015)). While we can relabel the multidimensional states into a onedimensional one, there is no natural ordering of states, not to say to argue or prove that some feature of the distribution of bids is higher in higher states. Therefore, our RSD ordering is particularly relevant when unobserved heterogeneity is multidimensional. In particular, we

\footnotetext{
${ }^{2}$ Krasnokutskaya (2012) studies such a problem using the deconvolution approach.
} 
extend the identification results of An (2016) by allowing for unobserved asymmetry in both bidders' preferences and private values.

\section{Related Literature}

For dealing with unobserved heterogeneity, there are other important methods besides the deconvolution method and the misclassification approach. See, e.g., Haile, Hong, and Shum (2003) and Guerre, Perrigne, and Vuong (2009) for using the number of bidders, Roberts (2013) for using observed reserve price and Armstrong (2013) for a partial identification approach. In English auctions where only winning bids are observed, Quint (2015) exploits exogenous participation and additively separability of unobserved heterogeneity to achieve identification.

Since Samuelson, economists have been studying comparative statics predictions (Athey (2002)). For instance, the theoretical auction literature has been interested in comparative statics in the stochastic dominance sense. See, e.g., Krishna (2009). In this paper, we introduce the concept of restricted stochastic dominance into auctions and study its properties. To the best of our knowledge, this seems to be new to the literature.

To learn about bidders' risk averseness, we consider the Guerre, Perrigne, and Vuong (2009) approach. For estimation methods based on this approach, see Zincenko (2014) and Kim (2015). For other approaches, see, e.g., Lu and Perrigne (2008) for exploiting the exogenous change of auction format, Campo, Guerre, Perrigne, and Vuong (2011) for a semiparametric method and Fang and Tang (2014) for using entry behaviors. Considering risk aversion and unobserved heterogeneity simultaneously is nontrivial. On the one hand, applying the misclassification approach requires a condition to order the resulting elements after eigenvalue decomposition. The usual approach is to find a monotonicity condition with respect to the state. Previous applications often had to make extra assumptions to ensure monotonicity. See, e.g., Gentry and Li (2014). Under risk aversion, it becomes more involved to find a feature of bid distribution that is monotone in the state. On the 
other hand, applying the results in Guerre, Perrigne, and Vuong (2009) requires finding two bid distributions that are derived from the same value distribution but under different competition levels. See Grundl and Zhu (2015) for some results on this. They rely on this monotonicity condition to match the two bid distributions resulting from the same value distribution but different numbers of bidders.

The rest of the paper is organized as follows. Section 2 describes the first-price auction model with risk aversion. Section 3 introduces the concept of restricted stochastic dominance and studies its properties in auctions. Moreover, we propose an ordering of a set of functions by defining its ordered tree. Section 4 applies these intermediate results in identification of the first-price auction model with discrete unobserved heterogeneity and risk neutrality. Section 5 applies the RSD ordering to identification of auction models with Beta distributed values, models with risk aversion, entry models and models with asymmetric bidders. Section 6 contains an empirical application of our method to data from U.S. Forest Service timber auctions. Section 7 concludes. Proofs omitted from the main text are collected in Appendices.

\section{The First-Price Auction Model}

We first introduce the first-price auction model in which bidders are risk averse. $I \geq 2$ symmetric bidders participate in a first-price auction with zero reserve price. They are potentially risk averse. Let $U(\cdot)$ be their utility function with $U(0)=0, U^{\prime}(\cdot)>0$ and $U^{\prime \prime}(\cdot)<0$. Conditioning on an auction-specific state $k \in \mathcal{K} \equiv\{1, \ldots, K\}$, their valuations are i.i.d. draws from the same distribution $F_{k}(\cdot)$ with support $\left[\underline{v}, \bar{v}_{k}\right]$. Denote $v_{k}(\cdot) \equiv F_{k}^{-1}(\cdot)$ as the corresponding quantile function. For exposition purpose, we treat the graph of $v_{k}(\cdot)$ as directioned while letting $\left(0, v_{k}(0)\right)$ and $\left(1, v_{k}(1)\right)$ be our starting point and ending point, respectively. For convenience, we focus on functions with the same starting point $v_{k}(0)=\underline{v}$. Suppose $v_{k}(\cdot)$ is continuously differentiable and their first-order derivatives are bounded away 
from both zero and infinity.

In a state- $k$ auction, a bidder with a valuation $v$ solves the following problem

$$
\max _{b} F_{k}\left(s_{k}^{-1}(b)\right)^{I-1} \cdot U(v-b),
$$

where $s_{k}^{-1}(\cdot)$ is the inverse of his/her optimal bidding strategy in state- $k$ auctions, and $F\left(s_{k}^{-1}(b)\right)^{I-1}$ is the chance of winning, i.e., the probability of his/her bid being the highest. Guerre, Perrigne, and Vuong (2009) study the identification of $F_{k}(\cdot)$ and $U(\cdot)$ when $k, I$ and the bid distribution $G_{k}(\cdot)$ are observed. To do so, they rewrite the equilibrium FOC in terms of observed bid quantile function ${ }^{3}$

$$
v_{k}(\alpha)=b_{k}(\alpha)+\lambda^{-1}\left(\frac{1}{I-1} \alpha b_{k}^{\prime}(\alpha)\right),
$$

where $\alpha \in[0,1], \lambda(\cdot)=U(\cdot) / U^{\prime}(\cdot)$, and $v_{k}(\cdot)$ and $b_{k}(\cdot)$ are the quantile functions of valuation and bid, respectively. Moreover, the boundary condition is $b_{k}(0)=v_{k}(0)=\underline{v}$.

Consider the case when the state $k$ is known to the econometrician. If the bidders are risk neutral (i.e., $U(x)=x$ ), Equation (1) shows the identification of $v_{k}(\cdot)$ with the knowledge of $b_{k}(\cdot)$ because $\lambda^{-1}(x)=x$. See Guerre, Perrigne, and Vuong (2000). On the other hand, if the bidders are potentially risk averse, Guerre, Perrigne, and Vuong (2009) shows that the model can always be rationalized by a first-price auction model where bidders have a CRRA or CARA utility function. In view of this, they propose to exploit an exogenous participation condition. Consider two sets of auctions, which are homogeneous (i.e., they have the same value distribution $\left.v_{k}(\cdot)\right)$ except having different numbers of bidders. Without loss of generality, assume that $2 \leq I_{1}<I_{2}$. Their idea is to exploit the exogenous variation

\footnotetext{
${ }^{3}$ While $s(\cdot)$ defines the mapping from value to bid, $b(\cdot)$ represents the bidding strategy as a function of the quantile of the value. The latter deals with different supports for comparative statics analysis. In some cases, it allows ordering the bidding behaviors of different bidders or in different auctions under weaker conditions.
} 
of the number of bidders $I$ and the compatibility condition:

$$
b_{k, I_{1}}(\alpha)+\lambda^{-1}\left(\frac{1}{I_{1}-1} \alpha b_{k, I_{1}}^{\prime}(\alpha)\right)=v_{k}(\alpha)=b_{k, I_{2}}(\alpha)+\lambda^{-1}\left(\frac{1}{I_{2}-1} \alpha b_{k, I_{2}}^{\prime}(\alpha)\right)
$$

where $b_{k, I}$ is the bid quantile function in state- $k$ auctions when the number of bidders is $I$. This condition implies the identification of $\lambda^{-1}(\cdot)$, which leads to the identification of $v_{k}(\cdot)$. Therefore, the model is identified with two sets of auctions under different competition levels.

If the state $k$ is unknown to the econometrician, we call it unobserved heterogeneity. It represents auction-specific characteristics observed by the bidders but unobserved by the econometrician. Hu, McAdams, and Shum (2013) consider identification of the component value distributions and the distribution of unobserved heterogeneity when bidders are risk neutral. Consider observing the bids from a set of homogeneous auctions where bidders are risk neutral. There are at least three bidders in each auction, say $\imath=i, j, \ell$.

They first discretize the bids by defining a partition of $R_{+}$into $K$ intervals, say $\mathcal{D}$ : $R_{+} \rightarrow\{1, \ldots, K\}$. Denote $D_{\imath}=\mathcal{D}\left(B_{\imath}\right)$ be the indicator function of the interval to which bidder $\imath$ 's bid $B_{\imath}$ belongs to, where $\imath=i, j, \ell$. To identify the auction model with unobserved heterogeneity, they assume a monotonicity condition and a full-rank condition.

Assumption 1 (UH monotonicity condition). There exists a known functional $M(\cdot)$ such that $M\left(v_{k}(\cdot)\right)$ is strictly increasing in $k$.

The existence and exact form of $M(\cdot)$ has to be determined case by case. They provide a list of examples where the maximum bid is increasing in the state. Other examples include the minimum bid and the mean equilibrium bid.

Assumption 2 (UH full-rank condition). There exists a discretization of bids such that the $K \times K$ matrix $L_{D_{i}, D_{j}}=\left[\operatorname{Pr}\left\{D_{i}=i^{\prime}, D_{j}=j^{\prime}\right\}\right]_{i^{\prime}, j^{\prime} \in\{1,2, \ldots, K\}}$ has rank $K$.

Hu, McAdams, and Shum (2013) provide a sufficient condition for both the UH monotonicity condition and the UH full-rank condition - the first-order stochastic dominance condition. For comparison, we rewrite it in terms of quantile functions. 
Definition 1 (First-Order Stochastic Dominance). $v_{k^{\prime}}(\cdot)$ first-order stochastically dominates $v_{k}(\cdot)$ if: (a) $v_{k}(\alpha) \leq v_{k^{\prime}}(\alpha), \forall \alpha \in[0,1]$, and (b) $\exists \alpha_{*} \in(0,1]$ such that $v_{k}\left(\alpha_{*}\right)<v_{k^{\prime}}\left(\alpha_{*}\right)$. .

Assumption 3 (Totality). For any two different states $k, k^{\prime} \in \mathcal{K}$, either $v_{k^{\prime}}(\cdot)$ first-order stochastically dominates $v_{k}(\cdot)$ or $v_{k}(\cdot)$ first-order stochastically dominates $v_{k^{\prime}}(\cdot)$.

Their method has two steps: first, identify the distribution of unobserved heterogeneity and the component bid distributions; second, apply Guerre, Perrigne, and Vuong (2000) to identify the underlying value distributions. While the second step is standard, they focus on the first one. Since bids are i.i.d. conditioning on the unobserved heterogeneity, the problem in the first step constitutes a finite mixture model with repeated measurements. Applying results from the measurement error literature (see $\mathrm{Hu}$ (2008)), they identify the component bid distributions up to permutation. Assumption 3 ensures a unique mapping from the anonymous distributions to the elements of $\mathcal{K}$.

\section{The Restricted Stochastic Dominance Ordering}

This section presents several important intermediate results. First, we introduce the concept of restricted stochastic dominance in auctions, which generalizes the first-order stochastic

dominance condition. Second, in first-price auctions, we show that the same relation of RSD passes from the value quantile functions to the bid quantile functions. Relying on these results, we propose an ordering of a set of value quantile functions and show that the corresponding set of bid quantile functions have the same ordering.

\subsection{Definitions}

In the auction literature, there are numerous results on first-order stochastic dominance passing from the value distribution functions to the bid distribution functions. See, e.g., Krishna (2009). However, in many cases, first-order stochastic dominance condition does not allow ranking two distributions under consideration because it is not a total ordering. 
To relax this condition, we introduce the concept of restricted stochastic dominance, which is particularly useful for situations where interests are focused on what happens below/above a certain threshold. In first-price auctions, the bidder cares about his/her valuation and bid only when he/she wins. Therefore, the bidder shall only consider the distribution below his/her valuation.

Definition 2 (Restricted Stochastic Dominance). $v_{k^{\prime}}(\cdot)$ dominates $v_{k}(\cdot)$ in the restricted sense if there exists an $x \in(0,1]$ such that: (a) $v_{k}(\alpha) \leq v_{k^{\prime}}(\alpha), \forall \alpha \in[0, x]$, and (b) $\exists \alpha_{*} \in$ $(0, x]$ such that $v_{k}\left(\alpha_{*}\right)<v_{k^{\prime}}\left(\alpha_{*}\right)$.

The concept of restricted stochastic dominance is introduced in Atkinson (1987) in the context of poverty measurement. See also Davidson and Duclos (2000) and Davidson and Duclos (2013). If one distribution first-order stochastic dominates another one, then the RSD condition is satisfied by letting $x=1$. Therefore, Assumption 4 is weaker than the firstorder stochastic dominance condition. While first-order stochastic dominance ordering is equivalent to expected utility ordering with an increasing utility function, the RSD ordering has a similar interpretation applying well-known results (e.g., Bawa (1975)). In particular, if $v_{2}(\cdot)$ dominates $v_{1}(\cdot)$ up to $x$, we can find a $z$ such that $F_{2}(\cdot)$ dominates $F_{1}(\cdot)$ up to $z$. Denote $\Pi_{k}(v)=\int_{\underline{v}}^{v} U(v-y) d F_{k}(y) . F_{2}(\cdot)$ dominates $F_{1}(\cdot)$ up to $z$ if and only if $\Pi_{1}(v) \leq \Pi_{2}(v)$ for all $v \in[0, z]$ and any differentiable and increasing function $U(\cdot)$ satisfying $U(0)=0$. To gain some intuition for this statement, consider ascending auctions where every bidder bids his/her private value. Conditioning on the bidder's private value $v$, his/her expected utility is higher in state- 2 ascending auctions than state- 1 ones for all $v \in[0, z]$.

Hereafter, we make a totality assumption.

Assumption 4 (Totality). For any two different states $k, k^{\prime} \in \mathcal{K}$, either $v_{k^{\prime}}(\cdot)$ dominates $v_{k}(\cdot)$ in the restricted sense or $v_{k}(\cdot)$ dominates $v_{k^{\prime}}(\cdot)$ in the restricted sense.

We can define a strict binary relationship between two functions at the infimum of the points below which there exist RSD relationships. 
Figure 1: Constructing An Ordered Tree from A Set of Distinct Functions

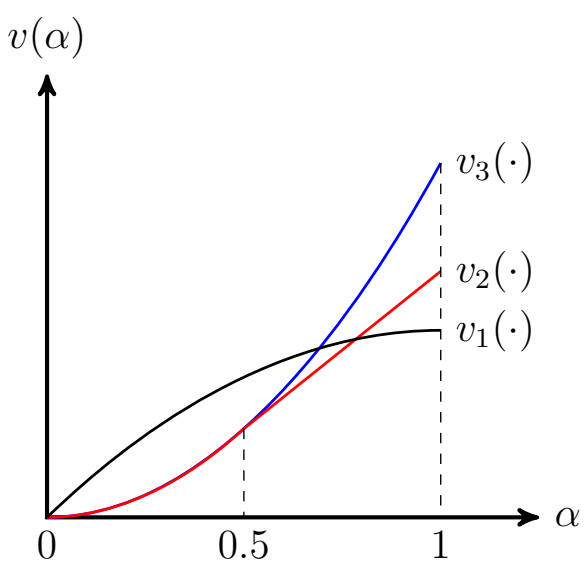

(a) Original Functions

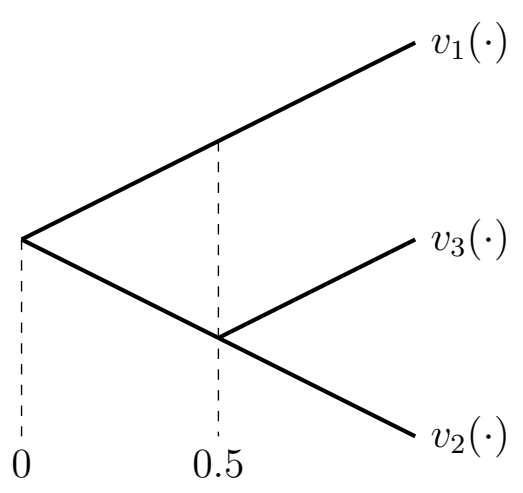

(b) Ordered Tree

Definition 3 (Splitting Point). We call $\alpha_{\dagger}$ the splitting point of $v_{k}(\cdot)$ and $v_{k^{\prime}}(\cdot)$ if $\alpha_{\dagger} \equiv$ $\inf \left\{x \in[0,1] \mid \forall \alpha \in[0, x], v_{k}(\alpha) \leq v_{k^{\prime}}(\alpha)\right.$, and $\left.\exists \alpha_{*} \in(0, x], v_{k}\left(\alpha_{*}\right)<v_{k^{\prime}}\left(\alpha_{*}\right)\right\}$.

We denote this relationship as $v_{k}(\cdot) \underset{\alpha_{\dagger}}{\prec} v_{k^{\prime}}(\cdot)$. It means that $v_{k}(\cdot)$ and $v_{k^{\prime}}(\cdot)$ coincide below $\alpha_{\dagger}$ and split apart right after $\alpha_{\dagger}$. By definition, for any $\epsilon>0$, there exists $x \in\left[\alpha_{\dagger}, \alpha_{\dagger}+\epsilon\right)$ such that $\forall \alpha \in[0, x], v_{k}(\alpha) \leq v_{k^{\prime}}(\alpha)$, and $\exists \alpha_{*} \in(0, x], v_{k}\left(\alpha_{*}\right)<v_{k^{\prime}}\left(\alpha_{*}\right)$. In fact, for any $\alpha \in[0, x], v_{k}(\alpha)<v_{k^{\prime}}(\alpha)$ implies $\alpha>\alpha_{\dagger}$. Otherwise, if $\alpha \leq \alpha_{\dagger}$ and $v_{k}(\alpha)<v_{k^{\prime}}(\alpha)$, by continuity of $v_{k}(\cdot)$ and $v_{k^{\prime}}(\cdot)$, we can find an $x^{\prime}<\alpha$ in the neighborhood of $\alpha$ such that $\forall \alpha \in\left[0, x^{\prime}\right], v_{1}(\alpha) \leq v_{2}(\alpha), \& \alpha_{*}^{\prime} \in\left(0, x^{\prime}\right], v_{k}\left(\alpha_{*}^{\prime}\right)<v_{k^{\prime}}\left(\alpha_{*}^{\prime}\right)$, a contradiction to the definition of $\alpha_{+}$.

We remark that once two functions split at $\alpha_{\dagger}$, we allow them to cross or touch again above $\alpha_{\dagger}$. Consider the example shown in Figure 1a, $v_{1}(\cdot)$ and $v_{2}(\cdot)$ split apart at $\alpha=0$, while $v_{2}(\cdot)$ and $v_{3}(\cdot)$ split apart at $\alpha=0.5$. The graph of $v_{1}(\cdot)$ crosses the graphs of $v_{2}(\cdot)$ and $v_{3}(\cdot)$ on $(0.5,1)$. Another example is $v_{k}(\alpha)=(2 \alpha)^{\gamma_{k}} / 2$, where $\gamma_{k} \in\{1 / 2,1,2\}$. The three functions split at 0 and cross again at $1 / 2$.

Next, we prove that this binary relationship is transitive. This result will be useful when we define the ordering of a set of value/bid quantile functions. 
Lemma 1 (Transitivity). If $v_{1}(\cdot) \underset{\alpha_{\dagger}}{\prec} v_{2}(\cdot)$ and $v_{2}(\cdot) \underset{\alpha_{\dagger}}{\prec} v_{3}(\cdot)$, then $v_{1}(\cdot) \underset{\alpha_{\dagger}}{\prec} v_{3}(\cdot)$.

Since we have already assumed totality under Assumption 4, transitivity provides a total ordering of the group of functions that split at the same point. Note that the set of splitting points is totally ordered by the usual less than or greater than relations. Therefore, there exist total orderings on the set $\left\{v_{k}(\cdot)\right\}_{k=1, \ldots, K}$, one of which we will define in Section 3.3.

In fact, Lemma 1 can be strengthened in the following way so that the RSD relationships directly imply a total ordering, which we call the RSD ordering. ${ }^{4}$

Lemma 2 (Transitivity). If $v_{1}(\cdot) \underset{\alpha_{\dagger}}{\prec} v_{2}(\cdot)$ and $v_{2}(\cdot) \underset{\alpha_{\dagger \dagger}}{\prec} v_{3}(\cdot)$, then $v_{1}(\cdot) \underset{\min \left\{\alpha_{\dagger}, \alpha_{\dagger \dagger}\right\}}{\prec} v_{3}(\cdot)$.

Intuitively, the bottom of the value distributions determines which dominates the others.

\subsection{Restricted Stochastic Dominance in Auctions}

To study RSD in the model we described in Section 2, we propose two alternative representations of the equilibrium FOC. Rearranging terms in Equation (1) gives

$$
b_{k}^{\prime}(\alpha)=\frac{I-1}{\alpha} \lambda\left(v_{k}(\alpha)-b_{k}(\alpha)\right)
$$

Therefore, $b_{k}(\cdot)$ satisfies the initial value problem if and only if it satisfies the integral problem

$$
b_{k}(\alpha)=\underline{v}+(I-1) \int_{0}^{\alpha} \frac{\lambda\left(v_{k}(x)-b_{k}(x)\right)}{x} d x
$$

Now we show that the same relation of RSD passes from the value quantile functions to the bid quantile functions. Consider an arbitrary $x \in(0,1]$.

Lemma 3. If $v_{1}(\alpha) \leq v_{2}(\alpha), \forall \alpha \in[0, x]$, then $b_{1}(\alpha) \leq b_{2}(\alpha), \forall \alpha \in[0, x]$.

This result is most obvious for the case of risk neutrality because we have an explicit

\footnotetext{
${ }^{4} \mathrm{I}$ thank a referee for this point.
} 
mapping from the value quantile function to the bid quantile function:

$$
b_{k}(\alpha)=(I-1) \alpha^{1-I} \int_{0}^{\alpha} v_{k}(x) x^{I-2} d x \text {. }
$$

The general case of risk aversion is less obvious due to a lack of explicit mapping. We include its proof in the Appendix. Similarly, we can show that $b_{1}(\alpha) \geq b_{2}(\alpha), \forall \alpha \in[0, x]$ if $v_{1}(\alpha) \geq$ $v_{2}(\alpha), \forall \alpha \in[0, x]$. Therefore, if $v_{1}(\alpha)=v_{2}(\alpha), \forall \alpha \in[0, x]$, then $b_{1}(\alpha)=b_{2}(\alpha), \forall \alpha \in[0, x]$.

Lemma 4. If $v_{1}(\alpha) \leq v_{2}(\alpha), \forall \alpha \in[0, x]$ and $v_{1}\left(\alpha_{*}\right)<v_{2}\left(\alpha_{*}\right)$, where $\alpha_{*} \in(0, x]$, then $b_{1}(\alpha)<b_{2}(\alpha), \forall \alpha \in\left[\alpha_{*}, x\right]$.

Lemma 4 states a striking feature. Specifically, if $v_{1}(\alpha) \leq v_{2}(\alpha)$ on $[0, x]$, not only the strict ordering passes from the value quantile functions to the bid quantile functions at the point of difference, it is maintained among the bid quantile functions till at least the cutoff point of the weakly dominance relation. An immediate implication is that the maximum bid $b_{k}(1)$ is increasing with respect to the state $k$ when Assumption 3 is true. Moreover, if $b_{2}(\cdot)$ dominates $b_{1}(\cdot)$ up to $x$, we can find a $z$ such that $G_{2}(\cdot) \equiv b_{2}^{-1}(\cdot)$ dominates $G_{1}(\cdot) \equiv b_{1}^{-1}(\cdot)$ up to $z$. Denote $\Pi_{k}(v)=\int_{\underline{v}}^{v} U(v-b) d G_{k}(b)$. We have $\Pi_{1}(v) \leq \Pi_{2}(v)$ for all $v \in[0, z]$. Intuitively, conditioning on the bidder's private value $v$, his/her expected utility is higher in state-2 first-price auctions than state-1 ones.

Together, Lemmas 3 and 4 imply that if $\left\{v_{k}(\cdot)\right\}_{k=1, \ldots, K}$ satisfies Assumption 4 , the induced $\left\{b_{k}(\cdot)\right\}_{k=1, \ldots, K}$ does as well. Therefore, there exist total orderings on the set $\left\{b_{k}(\cdot)\right\}_{k=1, \ldots, K}$, one of which is the RSD ordering.

Theorem 1. Under Assumption 4, the resulting $R S D$ orderings are the same for $\left\{v_{k}(\cdot)\right\}_{k=1,2, \ldots, K}$ and $\left\{b_{k}(\cdot)\right\}_{k=1,2, \ldots, K}$ in independent private value first-price auctions with risk averse bidders. Moreover, this property is invariant to the number of bidders I or the utility function $U(\cdot)$. 


\section{Discussion}

Since the lower bound of the value distribution $v_{k}(0)$ is also totally ordered by the usual less than or greater than relations, we can extend our discussion to allow $v_{k}(0)$ 's to differ across states. Moreover, the lower bound of the bid distribution equals the lower bound of the value distribution in symmetric first-price auctions. Therefore, the ordering of the lower bound also passes from the value quantile functions to the bid quantile functions.

While we use the probability $\alpha=0$ as our starting/reference point, it can be useful to use other starting/reference points in some applications. For instance, in asymmetric auctions, bidders take valuation draws from potentially different distributions. Using the probability $\alpha=1$ as our starting/reference point, we can find similar results on the ordering of the set of valuation distributions and the set of bid distributions when bidders are risk neutral. In this RSD ordering, the top of the value distributions determines which dominates the others. ${ }^{5}$

Moreover, simply for the purpose of ordering distributions, we can define an RSD relation when the relevant inequality hold over some other restricted range of the probability $\alpha$ rather than for an interval starting at 0 , say $[0, x]$. The RSD ordering can also be defined after some normalizations of the distributions, which include but are not limited to the usual "demean" exercise. An example is the normal distribution $\mathcal{N}\left(\mu, \sigma^{2}\right)$, where $\mu$ is the mean/median and $\sigma$ is the standard deviation. We can order a group of normal distributions first by the means/medians using the usual less than or greater than relations and second by the RSD relations on the right-hand side of the mean/median, i.e., $\alpha \in[0.5,1]$, which is equivalent to a lexicographical ordering of the pairs $\left(\mu_{k}, \sigma_{k}\right)$. In this RSD ordering, the part of the value distributions that is above and closest to the mean/median determines which distribution dominates the others. Another example is the Gumbel distribution $\mathcal{G}(\mu, \beta)$, whose mode is $\mu$ and variance is $\pi^{2} \beta^{2} / 6$. We can order a group of Gumbel distributions first by the modes and second by the RSD relations on the right-hand side of the mode. In this RSD ordering, the part of the value distributions that is above and closest to the mode determines which

\footnotetext{
${ }^{5}$ For completeness, we include these results in Appendix B.
} 
distribution dominates the others.

\subsection{An Intuitive Representation of the RSD ordering}

In this section, we propose an intuitive representation of the proposed RSD ordering. In particular, we propose a simple way to summarize how each function in a set of functions become distinguished from the rest. Relying on Lemmas 3 and 4, we know that this ordering is preserved by the mapping from value quantile function to bid quantile function.

Consider $v_{k}(\cdot)$, where $k \in \mathcal{K} \equiv\{1, \ldots, K\}$. We construct a "splitting path" $\mathcal{P}_{k}$ as follows:

(1) We find the smallest splitting point $\alpha_{1}$ where $v_{k}(\cdot)$ splits from some $v_{k^{\prime}}(\cdot)$ in group $\mathcal{K}$. That is,

$$
\alpha_{1}=\min _{k^{\prime} \neq k, k^{\prime} \in \mathcal{K}} \inf \left\{x \in[0,1] \mid \forall \alpha \in[0, x], v_{k}(\alpha) \leq v_{k^{\prime}}(\alpha), \& \exists \alpha_{*} \in[0, x], v_{k}\left(\alpha_{*}\right)<v_{k^{\prime}}\left(\alpha_{*}\right)\right\}
$$

By Lemma 1, we can rank all quantile functions based on their rankings in a neighborhood of $\alpha_{1}$, say $\left(\alpha_{1}, \alpha_{1}+\Delta_{1}\right]$. Let the smallest ranking be 1 . There is a group of functions that equal $v_{k}(\cdot)$ in $\left[0, \alpha_{1}+\Delta_{1}\right]$. Let this group be $\mathcal{K}_{1}$. All the functions in this group have the same ranking in $\left(\alpha_{1}, \alpha_{1}+\Delta_{1}\right]$, which we denote as $R_{1}$.

As shown in Figure 1a, if we consider $v_{2}(\cdot)$, we have $\alpha_{1}=0, R_{1}=1, \mathcal{K}_{1}=\{2,3\}$.

(2) We find the smallest splitting point $\alpha_{2}$ where $v_{k}(\cdot)$ splits from some $v_{k^{\prime}}$ in group $\mathcal{K}_{1}$. That is,

$$
\alpha_{2}=\min _{k^{\prime} \neq k, k^{\prime} \in \mathcal{K}_{1}} \inf \left\{x \in[0,1] \mid \forall \alpha \in[0, x], v_{k}(\alpha) \leq v_{k^{\prime}}(\alpha), \& \exists \alpha_{*} \in[0, x], v_{k}\left(\alpha_{*}\right)<v_{k^{\prime}}\left(\alpha_{*}\right)\right\}
$$

By Lemma 1, we can rank all quantile functions based on their rankings in a neighborhood of $\alpha_{2}$, say $\left(\alpha_{2}, \alpha_{2}+\Delta_{2}\right]$. Let the smallest ranking be 1 . There is a group of functions that equal $v_{k}(\cdot)$ in $\left[0, \alpha_{2}+\Delta_{2}\right]$. Let this group be $\mathcal{K}_{2}$. Denote the ranking of this group as $R_{2}$.

As shown in Figure 1a, if we consider $v_{2}(\cdot)$, we have $\alpha_{2}=0.5, R_{2}=1, \mathcal{K}_{2}=\{2\}$.

(3) We continue this process until $v_{k}(\cdot)$ is the only function in the group. 
We denote the list of pairs as $\mathcal{P}_{k} \equiv\left\{\left(\alpha_{1}, R_{1}\right), \ldots,\left(\alpha_{T_{k}}, R_{T_{k}}\right)\right\}$. We call $\mathcal{P}_{k}$ the splitting path of $v_{k}(\cdot)$. Note that $\mathcal{K}_{T_{k}}=\{k\}$. As shown Figure 1a, we have $\mathcal{P}_{1}=\{(0,2)\}, \mathcal{P}_{2}=$ $\{(0,1),(0.5,1)\}$ and $\mathcal{P}_{3}=\{(0,1),(0.5,2)\}$.

Definition 4 (Ordered Tree). An ordered tree is defined by the set of splitting paths $\mathcal{P} \equiv$ $\left(\mathcal{P}_{1}, \mathcal{P}_{2}, \ldots, \mathcal{P}_{K}\right)$.

This ordered tree can be represented as in Figure 1b. The junctions are located at the list of splitting points. The branches at each junction represent the rankings of functions with the bottom one being ranked the first. A splitting path is thus a path through the tree from the root to a leaf. It describes how a function became distinguished from the rest. Since we specify an ordering for the branches at each junction, the leaves (i.e., $\left.v_{k}(\cdot) \mathrm{s}\right)$ can be ordered from bottom to top.

Without loss of generality, we again denote the ordering in the ordered tree as $k=$ $1,2, \ldots, K$. Theorem 1 implies the following result.

Corollary 1. The resulting ordered trees and orderings are the same for $\left\{v_{k}(\cdot)\right\}_{k=1,2, \ldots, K}$ and $\left\{b_{k}(\cdot)\right\}_{k=1,2, \ldots, K}$ in first-price auctions.

The ordered tree summarizes the pairwise relations of RSD on a set of functions. Theorem 1 says that it provides a total ordering on a set of value distributions that is passed to the bid distributions and unaffected by the utility function and the number of bidders.

\section{Identification of Component Bid Distributions}

Without loss of generality, let $k=1,2, \ldots, K$ represent the RSD ordering defined by the set of primitive value quantile functions. In this section, we apply the eigenvalue decomposition as in $\mathrm{Hu}$, McAdams, and Shum (2013) to obtain the distributions of unobserved heterogeneity $p_{k}$ and the component bid distributions $G_{k}(\cdot)$. 
Theorem 2. Under Assumptions 2 and 4, the distribution of unobserved heterogeneity $p_{k}$ and the component bid distributions $G_{k}(\cdot)$ are identified if $I \geq 3$.

Proof. We follow closely the identification procedure in Hu, McAdams, and Shum (2013). Consider three bidders $\ell, i$ and $j$. We define a discretization of bids as a monotone onto mapping $\mathcal{D}: R_{+} \rightarrow\{1, \ldots, K\}$, which partition the bid-space into $K$ intervals. Denote $D_{\imath}=\mathcal{D}\left(B_{\imath}\right)$ be the interval to which bidder $i$ 's bid belongs, where $\imath=i, j$. Fix the value of bidder $\ell$ 's bid, say $\breve{b}_{\ell}$. Define the following matrices:

$$
\begin{aligned}
L_{D_{i} \mid K} & =\left[g_{D_{i} \mid K}\left(i^{\prime} \mid k\right)\right]_{i^{\prime}, k=1,2, \ldots, K}, \\
L_{D_{j}, D_{i}} & =\left[g_{D_{j}, D_{i}}\left(j^{\prime}, i^{\prime}\right)\right]_{j^{\prime}, i^{\prime}=1,2, \ldots, K}, \\
L_{D_{i}, \breve{b}_{\ell}, D_{j}} & =\left[g_{D_{i}, B_{\ell}, D_{j}}\left(i^{\prime}, \check{b}_{\ell}, j^{\prime}\right)\right]_{i^{\prime}, j^{\prime}=1,2, \ldots, K}, \\
D_{\check{b}_{\ell} \mid K} & =\operatorname{diag}\left\{\left[g_{B_{\ell} \mid K}\left(\check{b}_{\ell} \mid k\right)\right]_{k=1,2, \ldots, K}\right\},
\end{aligned}
$$

where $K$ represents the random variable of unobserved heterogeneity with a slight abuse of notation, $g_{D_{i} \mid K}\left(i^{\prime} \mid k\right)$ is the conditional probability of observing $D_{i}=i^{\prime}$ at state $k, g_{D_{j}, D_{i}}\left(j^{\prime}, i^{\prime}\right)$ is the probability of observing $\left(D_{j}, D_{i}\right)=\left(j^{\prime}, i^{\prime}\right), g_{D_{i}, B_{\ell}, D_{j}}\left(i^{\prime}, \check{b}_{\ell}, j^{\prime}\right)$ is the joint density of $\left(D_{i}, B_{\ell}, D_{j}\right)$ at $\left(i^{\prime}, \breve{b}_{\ell}, j^{\prime}\right)$, and $g_{B_{\ell} \mid K}\left(\breve{b}_{\ell} \mid k\right)$ is the conditional bid density at state $k$.

Applying the results in $\mathrm{Hu}$ (2008), $\mathrm{Hu}$, McAdams, and Shum (2013) obtain their key identification equation:

$$
L_{D_{i}, \check{b}_{\ell}, D_{j}}\left(L_{D_{j}, D_{i}}^{T}\right)^{-1}=L_{D_{i} \mid K} D_{\check{b}_{\ell} \mid K} L_{D_{i} \mid K}^{-1},
$$

whose left-hand side is identified from data and the terms in the right-hand side can be obtained through eigenvalue decomposition. Following the same lines, the $K$ eigenvectors are uniquely determined. However, the ordering is still arbitrary. That is, $L_{D_{i} \mid K}$ is identified up to permutation of its columns.

We now depart from Hu, McAdams, and Shum (2013) by swapping their last two steps. 
In particular, we fix an eigenvector matrix $\overline{L_{D_{i} \mid K}}=L_{D_{i} \mid K} Q$, which is a matrix generated by interchanging columns of the true eigenvector matrix $L_{D_{i} \mid K} \cdot Q$ is an unknown elementary matrix generated by interchanging columns of the identity matrix.

We now identify

$$
\overline{D_{\check{b}_{\ell} \mid K}}={\overline{L_{D_{i} \mid K}}}^{-1}\left[L_{D_{i}, \check{b}_{\ell}, D_{j}}\left(L_{D_{j}, D_{i}}^{T}\right)^{-1}\right] \overline{L_{D_{i} \mid K}}
$$

which is is a diagonal matrix. Therefore, by varying the value of $\breve{b}_{\ell}$, we identify the component bid density functions (and quantile functions) up to permutation because

$$
\overline{D_{\check{b}_{\ell} \mid K}}=\left(L_{D_{i} \mid K} Q\right)^{-1}\left[L_{D_{i}, \check{b}_{\ell}, D_{j}}\left(L_{D_{j}, D_{i}}^{T}\right)^{-1}\right] L_{D_{i} \mid K} Q=Q^{-1} D_{\check{b}_{\ell} \mid K} Q
$$

Note that the set of these "anonymous" component bid quantile functions is precisely the same as the set of primitive bid quantile functions. Thus, we can construct the same ordered tree using the former and the latter. Following Corollary 1 , the $k$ th function in the set of primitive value quantile functions is ranked the $k$ th in the set of identified "anonymous" functions according to the RSD ordering. Therefore, it pins down $D_{\check{b}_{\ell} \mid K}$ and hence determines the matrix $Q$. Moreover, $L_{D_{i} \mid K}=\overline{L_{D_{i} \mid K}} Q^{-1}$ is identified. The rest of the proof is identical with that of $\mathrm{Hu}$, McAdams, and Shum (2013).

Theorem 2 provides the component bid distributions $G_{k}(\cdot)$ and the distribution of unobserved heterogeneity $p_{k}$. If bidders are risk neutral, the component value distributions $F_{k}(\cdot)$ are identified according to Guerre, Perrigne, and Vuong (2000). In particular, we have the following $\mathrm{FOC}$ in quantile terms:

$$
v_{k}(\alpha)=b_{k}(\alpha)+\frac{1}{I-1} \alpha b_{k}^{\prime}(\alpha)
$$

Therefore, the symmetric first-price auction model with discrete unobserved heterogeneity and risk neutrality is fully identified. 


\section{A Sufficient Condition for Assumption 2}

This subsection introduces a sufficient condition for the rank condition Assumption 2.

Theorem 3. Assumption 2 is satisfied if the component bid distribution functions are linearly independent.

This condition has been used in An (2016) for identifying the number of types $K$. Following his paper, one could also describe the linear independence condition in terms of primitives: the functions $\left\{F_{1}\left(s_{1}^{-1}(\cdot)\right), \ldots, F_{K}\left(s_{K}^{-1}(\cdot)\right)\right\}$ are linearly independent, where $s_{k}^{-1}(\cdot)$ is the inverse bidding strategy in state- $k$ auctions defined by the model primitives $\left\{U(\cdot), F_{k}(\cdot)\right\}$.

Recall that the first-order stochastic dominance condition is sufficient for both the UH monotonicity condition and the UH full-rank condition. See Hu, McAdams, and Shum (2013). A key step in their proof is to show that the maximum bid is increasing in the state $k$, which implies that the component bid distribution functions are linearly independent. ${ }^{6}$

\section{A Sufficient Condition for Assumption 4}

While our RSD condition is weaker than the first-order stochastic dominance condition, it is interesting to understand the generality of our condition. To this end, we now describe another sufficient condition for Assumption 4.

Theorem 4. Assumption 4 is satisfied if $\left\{v_{k}(\cdot)\right\}_{k=1, \ldots, K}$ are analytic.

An example of a non-analytic function is $f(x)=x^{4} \sin (1 / x)$, where $x \in[0,1]$. See Figure 2 for its graph on $[0,0.01]$. In fact, for any $x \in(0,1]$, we can always find a change of sign in $(0, x]$. If this function were the difference of the two value quantile functions, it is obvious that it is continuously differentiable but there exists no splitting point. For instance, let $v_{1}(\alpha)=\alpha^{2}$ and $v_{2}(\alpha)=\alpha^{2}+\alpha^{4} \sin (1 / \alpha)$. Figure 2 shows that $v_{1}(\cdot)$ and $v_{2}(\cdot)$ are strictly increasing and hence valid quantile functions but they violate Assumption 4.

\footnotetext{
${ }^{6}$ In fact, if $\sum_{k=1}^{K} \gamma_{k} G_{k}(b)=0$, then $\gamma_{k}=0, \forall k \in \mathcal{K}$. To see this, consider an arbitrary $b \in\left(\bar{b}_{K-1}, \bar{b}_{K}\right)$. Since $\sum_{k=1}^{K} \gamma_{k} G_{k}(b)=\gamma_{K} G_{K}(b)=0$, we have $\gamma_{K}=0$. Similarly, we can show that $\gamma_{k}=0$ for all $k=K-1, \ldots, 1$ by considering a bid $b$ in $\left(\bar{b}_{K-2}, \bar{b}_{K-1}\right), \ldots,\left(\bar{b}_{1}, \bar{b}_{2}\right)$ sequentially.
} 
Note that analytic functions are infinitely differentiable. They include all elementary functions such as the polynomial functions, the exponential functions and the trigonometric functions. Moreover, the Weierstrass Approximation Theorem says that any continuous function on a bounded interval can be uniformly approximated by polynomial functions, which are analytic. For assuming analytic functions to achieve identification in econometrics, See, e.g., Fox, il Kim, Ryan, and Bajari (2012) in a random coefficients logit model, Quint (2015) in English auctions, Fox and Gandhi (2016) in a multinomial choice model, and Aryal (2016) in an adverse selection model.

Nevertheless, Assumption 4 does allow some non-analytic functions. For instance, $v_{1}(\alpha)=$ $\alpha, v_{2}(\alpha)=\alpha+\exp (-1 / \alpha)$, if $\alpha \in(0,1]$ and $v_{1}(0)=v_{2}(0)=0$.

Figure 2: A non-analytic function: $v_{2}(\alpha)-v_{1}(\alpha)=\alpha^{4} \sin (1 / \alpha)$
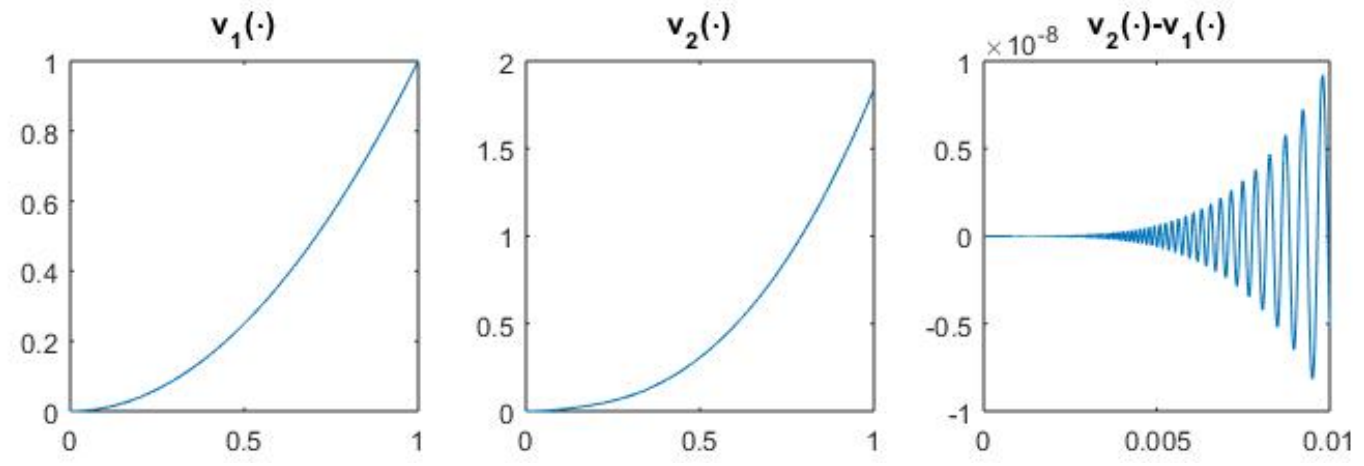

\section{Applications}

In this section, we demonstrate several applications that show how our relaxation of the first-order stochastic dominance condition further expands the breadth of applicability of the $\mathrm{Hu}$, McAdams, and Shum (2013) misclassification approach. In particular, we apply the RSD ordering in identification of several auction models with unobserved heterogeneity: (a) values are i.i.d. draws from Beta distributions; (b) nonparametric utility function; (c) affiliated-signal entry models; (d) asymmetric bidders; (e) asymmetry in both preferences and private values. 


\subsection{Beta Distribution}

An immediate application of our results is on identification of auctions when values are from Beta distributions. With a slight abuse of notation, the Beta distribution $\mathcal{B}(\alpha, \beta)$ is a family of continuous distributions defined on $[0,1]$ and parameterized by two positive shape parameters $\alpha, \beta>0$. Its density function is

$$
f(v ; \alpha, \beta)=\frac{v^{\alpha-1}(1-v)^{\beta-1}}{\int_{0}^{1} x^{\alpha-1}(1-x)^{\beta-1} d x},
$$

if $v \in[0,1], f(v ; \alpha, \beta)=0$ otherwise. It is a flexible distribution that has bounded support. In fact, any continuous distribution function on $[0,1]$ can be approximated by a convex combination of Beta distributions. See Diaconis and Ylvisaker (1985). It is known that this family of distributions cannot be ranked by the first-order stochastic dominance relations. We now show that they can be ranked by the RSD relations.

Lemma 5. Any set of distinct Beta distributions satisfies Assumption 4.

In fact, all the Beta distributions split at $\alpha_{\dagger}=0$. Consider two different Beta distributions defined by $\left(\alpha_{1}, \beta_{1}\right)$ and $\left(\alpha_{2}, \beta_{2}\right)$, respectively. It is easy to show that if $v \rightarrow 0$, then the difference $\log f_{2}(v)-\log f_{1}(v)$ converges to (1) $-\infty$ if $\alpha_{1}<\alpha_{2},(2)+\infty$ if $\alpha_{1}>\alpha_{2}$, (3) a positive number if $\alpha_{1}=\alpha_{2}$ and $\beta_{1}<\beta_{2}$, and (4) a negative number if $\alpha_{1}=\alpha_{2}$ and $\beta_{1}>\beta_{2}$. This leads to a lexicographical ordering based on the RSD relation: first we rank the two $\alpha$ 's, the larger $\alpha$ is, the higher ranked the distribution is; in case the two $\alpha$ 's are the same, we rank $\beta$ 's, the larger $\beta$ is, the lower ranked the distribution is. Figure 3 shows the quantile functions of Beta distributions in $[0,1]$ and $[0,0.01]$ when $\alpha, \beta \in\{0.5,1,2\}$. While these functions cannot be ordered on $[0,1]$, they do in a neighborhood of 0 .

Theorem 5. Under Assumptions 2, if $I \geq 3$ and the values are i.i.d. draws from Beta distributions $F\left(\cdot ; \alpha_{k}, \beta_{k}\right)$ conditioning on $k \in\{1, \ldots, K\}$, the symmetric first-price auction model with discrete unobserved heterogeneity and risk neutrality is identified. 
Figure 3: The Quantile Functions of Beta Distributions: $\alpha, \beta \in\{0.5,1,2\}$

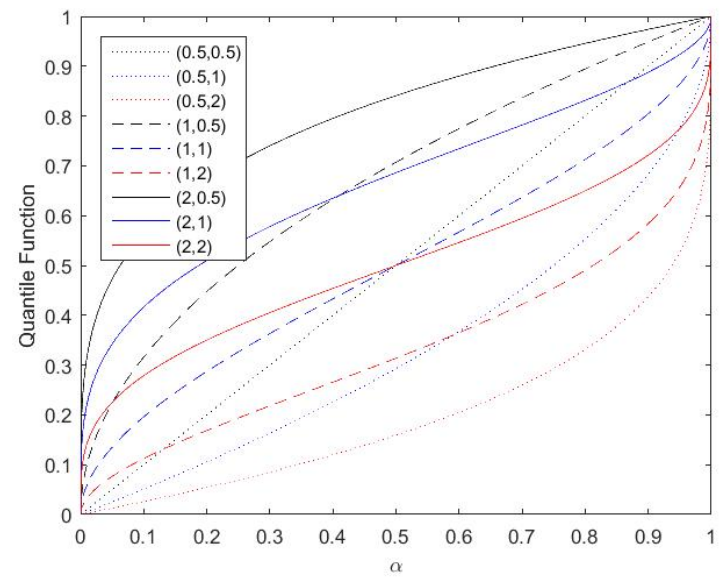

(a) Probability $\alpha \in[0,1]$

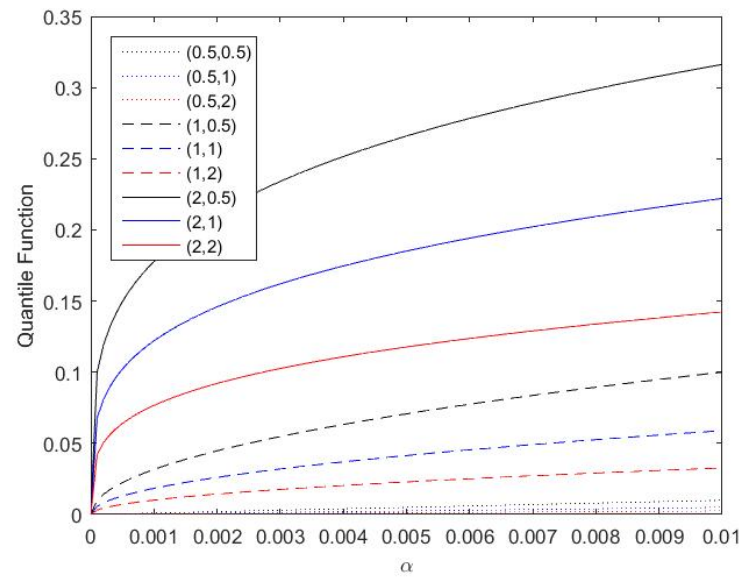

(b) Probability $\alpha \in[0,0.01]$

\subsection{Guerre, Perrigne, and Vuong (2009): Risk Aversion}

Theorem 1 says that the ordered trees are the same for the induced bid distributions regardless of the utility function and the number of bidders. This result is particularly useful to identify auction models with discrete unobserved heterogeneity $k$ and risk aversion (i.e., $\left.U^{\prime \prime}(\cdot)<0\right)$ in the Guerre, Perrigne, and Vuong (2009) framework. To identify both the utility and the value distribution functions, the Guerre, Perrigne, and Vuong (2009) method requires two sets of auctions which have different numbers of bidders and assumes exogenous participation. In this section, we show how to adjust their exogenous participation approach and the two-step method of Hu, McAdams, and Shum (2013) to identify the model when the state $k$ is not known to the econometrician by applying Theorem 1 .

In particular, we consider two sets of auctions with unobserved heterogeneity $k$, which have $I_{1}$ and $I_{2}$ bidders, respectively. We observe the joint distribution of three bids $\left(b_{i}, b_{j}, b_{\ell}\right)$ conditioning on $I=I_{1}, I_{2}$. We consider the identification of the distributions of unobserved heterogeneity $p_{k, I}$, the utility function $U(\cdot)$, as well as the component value distributions $F_{k, I}(\cdot)$.

If bidders are risk averse, we need to deal with the unknown utility function $U(\cdot)$. We follow Guerre, Perrigne, and Vuong (2009) to exploit the exogenous participation restriction. 
Assumption 5. $F_{k, I_{1}}(\cdot)=F_{k, I_{2}}(\cdot)=F_{k}(\cdot)$.

Note that we allow $p_{k, I_{1}}$ and $p_{k, I_{2}}$ to differ. Conditioning on the number of bidders $I \in\left\{I_{1}, I_{2}\right\}$, we can apply Theorem 2 to obtain the distribution of unobserved heterogeneity $p_{k, I}$, the component bid distributions $G_{k, I}(\cdot)$ and the corresponding bid quantile functions $b_{k, I}(\cdot)$. Identification of $\lambda(\cdot)$ is achieved if we can match the two bid quantile functions that are derived from the same unobserved heterogeneity $k$ but with different number of bidders, say $3 \leq I_{1}<I_{2}$.

The difficulty is how to match the bid quantile functions in the two lists. The reason is that with the same unobserved heterogeneity, the resulting component bid distributions are different due to different competition levels $I_{1}$ and $I_{2}$. If bidders are risk neutral, these two bid distributions are related through a known mapping due to exogenous participation ${ }^{7}$ :

$$
b_{k, I_{2}}(\alpha)=\frac{I_{2}-1}{I_{1}-1}\left[b_{k, I_{1}}(\alpha)+\left(I_{1}-I_{2}\right) \alpha^{1-I_{2}} \int_{0}^{\alpha} b_{k, I_{1}}(x) x^{I_{2}-2} d x\right] .
$$

With risk averse bidders, it is unclear how to make a similar connection before identifying bidders' utility function. In other words, we are not sure whether the difference between two bid quantile functions is due to different competition levels or unobserved heterogeneity.

We now show how to deal with this issue using our intermediate results. While we are not able to match bid distributions through a known functional, the corresponding bid quantile functions share the same ordered tree, which is inherited from the same set of value quantile functions and unaffected by the number of bidders.

Applying Theorem 2, we first identify the component bid quantile functions $G_{k, I}$ conditioning on the number of bidders $I=I_{1}, I_{2}$, respectively. This gives two ordered trees, $\mathcal{P}^{I_{1}}$ and $\mathcal{P}^{I_{2}}$, for the two samples of auctions, respectively. The two trees are identical. Second, we apply Theorem 1 to match these bid quantile functions into pairs. The idea is that two bid quantile functions are generated by the same value quantile function if they are the same

\footnotetext{
${ }^{7}$ See Appendix A.5 for its proof.
} 
leaves on the two identical ordered trees. Finally, we can apply Guerre, Perrigne, and Vuong (2009) to identify risk aversion by exploiting the compatibility condition:

$$
b_{k, I_{1}}(\alpha)+\lambda^{-1}\left(\frac{1}{I_{1}-1} \alpha b_{k, I_{1}}^{\prime}(\alpha)\right)=b_{k, I_{2}}(\alpha)+\lambda^{-1}\left(\frac{1}{I_{2}-1} \alpha b_{k, I_{2}}^{\prime}(\alpha)\right) .
$$

Therefore, the model is identified with two sets of auctions under different competition levels.

Theorem 6. Under Assumptions 2, 4 and 5, the symmetric first-price auction model with discrete unobserved heterogeneity and risk aversion is identified if $I_{2}>I_{1} \geq 3$.

In a related paper, Grundl and Zhu (2015) show that the deconvolution method applies to nonparametric utility functions if unobserved heterogeneity enters valuations additively, but only to CRRA utility functions if it enters multiplicatively. While the $\mathrm{Hu}$, McAdams, and Shum (2013) approach requires more bids than the deconvolution method, Theorem 6 says that their misclassification approach is robust to the utility function. The intuition comes from the fact that the independence of bids can be inherited from independent values, while the multiplicative separability of bids may not necessarily be inherited from multiplicatively separable values.

\section{Discussion}

Note that Theorem 2 identifies $2 K$ functions (i.e., $K$ component bid distributions for each $I$ ), but the number of unkonwn primitive functions is $1+K$ (i.e., the common utility function $U(\cdot)$ and the value distributions $\left.F_{k}(\cdot)\right)$. Obviously, this model is overidentified. An interesting extension is to allow the utility function be state-specific, say $U_{k}(\cdot)$. The model becomes more general and its primitives are $\left\{U_{k}(\cdot), F_{k}(\cdot), p_{k, I_{1}}, p_{k, I_{2}}\right\}$.

To identify the new model, complications arise due to the interaction of $U_{k}(\cdot)$ and $F_{k}(\cdot)$. Obtaining comparative statics is difficult using the usual stochastic dominance notions. To make it worse, since $U_{k}(\cdot)$ and $F_{k}(\cdot)$ jointly determine $b_{k}(\cdot)$, the same RSD ordering may not pass from the value quantile functions to the bid quantile functions. 
Despite the difficulties, the notion of RSD is still useful in terms of identifying ordering because it only requires comparing function locally. One possible identification strategy is to use a point where the interaction of $U_{k}(\cdot)$ and $F_{k}(\cdot)$ disappears. In particular, we focus on the probability $\alpha=0$ and show that $v_{k}^{\prime}(0)=\frac{I}{I-1} b_{k}^{\prime}(0)$, where $I=I_{1}, I_{2}$ and $k \in \mathcal{K}$. Therefore, the same RSD ordering passes from the value quantile functions to the bid quantile functions. In sum, the notion of RSD leads to a simple identification assumption.

Assumption 6. $v_{1}^{\prime}(0)<v_{2}^{\prime}(0)<\cdots<v_{K}^{\prime}(0)$.

Assumption 6 says that the splitting point is 0 for any two value quantile functions $v_{k}(\cdot)$ and $v_{k^{\prime}}(\cdot)$. Many families of distribution functions with bounded supports satisfy this condition. For instance, the Beta distribution, the uniform distribution and the two-sided power distribution. We summarize the results in the following theorem.

Theorem 7. Under Assumptions 2, 4, 5 and 6, the symmetric first-price auction model with discrete unobserved heterogeneity and state-specific risk aversion is identified if $I_{2}>I_{1} \geq 3$.

\subsection{Gentry and Li (2014): Affiliated-Signal Model}

Gentry and Li (2014) study identification of the Affiliated-Signal model under risk neutrality, in which potential bidders have private values $v$, observe imperfect signals $s$ of their true values prior to entry, choose whether to undertake a costly entry process and learn their exact values and submit bids. Let $F(v, s \mid I, z)$ be the joint distribution of private value and signal conditioning on the number of bidders $I$ and a continuous cost shifter $z$, and $c(I, z)$ be the entry cost function. With two excludable variations $F(v, s \mid I, z)=F(v, s)$ and $c(I, z)=c(z)$, they show that the conditional value distribution $F(\cdot \mid s)$ and the entry cost function $c(z)$ is point-identified when $z$ is continuous. ${ }^{8}$

They generalize their results to accommodate unobserved heterogeneity $k$ by introducing the following assumptions: (1) conditional independence $\left(v_{i}, s_{i}\right) \perp\left(v_{j}, s_{j}\right) \mid k ;(2)$ conditional excludability: $F(v, s \mid I, z, k)=F(v, s \mid k)$ and $c(I, z, k)=c(z ; k) ;$ and $(3)$

\footnotetext{
${ }^{8}$ They have interesting results on partial identification with incomplete variation, which we omit here.
} 
Assumption 7 (Gentry and Li (2014)). 1. Stochastic ordering: $F\left(v \mid s, k^{\prime}\right) \leq F(v \mid s, k)$ if $k^{\prime} \geq k$; 2. Entry ordering: for all $(I, z)$, the entry threshold $s^{*}(I, z ; k)$ is decreasing in $k$.

While Assumptions (1) and (2) are standard and both the stochastic ordering and entry ordering are intuitive, the orderings are assumed to ensure that the CDF of "realized bids" $G_{w}^{*}$ can be indexed with respect to $k$.

$$
G_{w}^{*}(W \mid I, z ; k)=\left\{\begin{array}{l}
s^{*}(I, z ; k)+\left[1-s^{*}(I, z ; k)\right] G_{b}^{*}(b \mid I, z ; k), \text { if } W>0 \\
s^{*}(I, z ; k), \text { if } W=0
\end{array}\right.
$$

where $W_{i}=B_{i}$ if $i$ enters and $W_{i}=0$ otherwise. $s^{*}(I, z ; k)$ is the entry threshold. The authors remark that the mixed random variables $\left(W_{1}, \ldots, W_{I}\right)$ are conditionally independent given $k$ and stochastically increasing in $k$ under Assumption 7 .

The identification proceeds as follows: Given $I \geq 3$ and $z$, applying the $\mathrm{Hu}$, McAdams, and Shum (2013) results, $G_{w}^{*}\left(W_{i} \mid I, z ; k\right)$ and $p_{k}(I, z)$ are identified up to permutation from the joint distribution $G_{W}\left(W_{1}, \ldots, W_{I} \mid I, z\right)$. Stochastic ordering of $G_{w}^{*}\left(W_{i} \mid I, z ; k\right)$ ensures a unique mapping from the anonymous distributions to the elements of $\mathcal{K}$ for every pair $(I, z)$.

In fact, the monotonicity of the entry threshold is sufficient for ordering unobserved heterogeneity conditioning on every pair $(I, z)$. To see this, we construct an ordered tree using the corresponding quantile function of $\left\{G_{w}^{*}\left(W_{i} \mid I, z ; k\right)\right\}_{k=1, \ldots, K}$. Since the quantile function remains 0 on $\left[0, s^{*}(I, z ; k)\right]$ and jumps to a positive number afterward, the RSD ordering of $G_{w}^{*}\left(W_{i} \mid I, z ; k\right)$ is increasing with respect to $k$. Therefore, allowing for unobserved heterogeneity, the Gentry and Li (2014) results hold under a smaller set of assumptions: (1) conditional independence; (2) conditional excludability; and (3) entry ordering.

\section{Discussion}

In general, by introducing the covariate $z$, we have a problem of identifiability of finite mixture models with covariates, which suffers from a "label switching" problem. To deal 
with it, further assumptions are usually made. For instance, to identify auction models with asymmetric anonymous bidders and covariates, Lamy (2012) adopts a similar strategy by assuming that any two bid distributions are either equal or can be strictly ordered according to first-order stochastic dominance. That is, either $G(\cdot \mid z, k)$ and $G\left(\cdot \mid z, k^{\prime}\right)$ are the same for any $z$ or $G(b \mid z, k)<G\left(b \mid z, k^{\prime}\right)$ for any $b$ and any $z$. In view of our results, identification can be achieved by assuming that there exists a known functional $\mathcal{M}$ that yields the same ordering when applied to the set of value functions $\{G(\cdot \mid z, k)\}_{k=1, \ldots, K}$ for every $z$.

For instance, consider symmetric first-price auctions with auction-specific covariates $z$ and unobserved heterogeneity $k$, where private values are i.i.d. draws from the Beta distribution with shape parameters $(\alpha(z, k), \beta(z, k))$. The analyst observes repeated measurements, i.e. three bids per auction $\left(b_{1}, b_{2}, b_{3}\right)$, which has joint cumulative distribution

$$
G\left(b_{1}, b_{2}, b_{3} \mid z\right)=\sum_{k=1}^{K} p_{k \mid z} \times \Pi_{i=1}^{3} G_{\mathcal{B}}\left(b_{i} ; \alpha(z, k), \beta(z, k)\right),
$$

where $p_{k \mid z}$ is the conditional probability of unobserved heterogeneity, $(\alpha(z, k), \beta(z, k))$ are the shape parameters conditioning on $(z, k)$, and $G_{\mathcal{B}}(\cdot ; \alpha(z, k), \beta(z, k))$ is the corresponding bid distribution. In view of our results in Subsection 5.1, we provide a sufficient condition for nonparametric identification of the model primitives $\left\{\alpha(\cdot, \cdot), \beta(\cdot, \cdot), p_{k \mid z}\right\} .{ }^{9}$

Corollary 2. Under Assumptions 2, if $I \geq 3, \alpha(z, k)$ is strictly monotone in $k$, and the values are i.i.d. draws from Beta distributions $F(\cdot ; \alpha(z, k), \beta(z, k))$ conditioning on $(z, k)$, the symmetric first-price auction model with auction-specific covariates $z$, discrete unobserved heterogeneity $k$ and risk neutrality is identified.

\subsection{An (2016): Asymmetric Bidders}

An (2016) studies identification of first-price auction models where bidders' values are asym-

\footnotetext{
${ }^{9}$ While we constrain ourselves to discrete unobserved heterogeneity in this paper, the RSD ordering can be applied in the continuous case. See, e.g., Hu and Schennach (2008). We leave it for future research.
} 
metrically distributed. ${ }^{10}$ He considered a model with $I$ risk-neutral bidders whose private values are independent draws from $F_{1}(\cdot), \ldots, F_{K}(\cdot)$, which are CDFs with the same support $[\underline{v}, \bar{v}]$. Each bidder is one of the $K$ types. Let $\tau_{i}$ denote bidder $i$ 's type known to all the bidders but unknown to the econometrician. Bidder $i$ 's bidding strategy is a mapping from his/her value to his/her bid, i.e., $s_{i}(\cdot, \cdot):[\underline{v}, \bar{v}] \times\{1, \ldots, K\} \rightarrow[\underline{v}, \bar{v}]$. An (2016) assumes that each bidder participates several auctions and the type is invariant, so the econometrician observes the bidders' identity and the joint distribution $G\left(b_{\ell}, b_{\ell^{\prime}}, b_{\ell^{\prime \prime}}\right)$.

Applying the results in $\mathrm{Hu}$ (2008), he identifies the type specific bid distribution $G_{k}(\cdot)$ and then the value distribution $F_{k}(\cdot)$ under Assumption 3. In view of our results, his results hold under a weaker condition Assumption 4.

\subsection{Multidimensional Unobserved Heterogeneity: Asymmetry in Both Preferences and Private Values}

Now we generalize An (2016)'s results to allow for asymmetry in both preferences and private values. That is, bidders differ not only in their value distributions but also in their utility functions. For simplicity, we assume a CRRA utility function for the bidders $U(x)=x^{\theta}$, where $\theta \in\left\{\theta_{H}, \theta_{L}\right\}$. Assume $0<\theta_{L}<\theta_{H} \leq 1$ so that type- $\theta_{L}$ bidders are more risk averse than type- $\theta_{H}$ bidders. Moreover, we assume that the econometrician knows the values of $\theta_{L}$ and $\theta_{H}$ for simplicity of exposition. ${ }^{11}$

Each bidder is one of the $K$ types whose private values are independent draws from $F_{1}(\cdot), \ldots, F_{K}(\cdot)$. Following Guerre, Perrigne, and Vuong (2000) and Flambard and Perrigne (2006), the FOC can be rewritten as (see also Appendix B)

$$
s_{i}^{-1}(b)=b+\frac{\theta_{i}}{\sum_{j \neq i} g_{j}(b) / G_{j}(b)},
$$

\footnotetext{
${ }^{10} \mathrm{An}(2016)$ also studies identification of the first-price auction model with non-equilibrium beliefs. We omit the discussion here because ordering follows from model restrictions.

${ }^{11}$ To identify these two parameters, we can introduce exogenous variations in the competition level $I$ as in Guerre, Perrigne, and Vuong (2009). See Subsection 5.2.
} 
where $g_{i}(\cdot)$ and $G_{i}(\cdot)$ are the bid density and distribution functions of bidder $i=1, \ldots, I$. Since $F_{i}(\cdot)=G_{i}\left(s_{i}(\cdot)\right)$, identifying $s_{i}^{-1}(\cdot)$ and $G_{i}(\cdot)$ implies the identification of $F_{i}(\cdot)$.

Note that bidder $i$ is of type $k \in\{1, \ldots, K\}$ and $\theta \in\left\{\theta_{H}, \theta_{L}\right\}$. While the bidders' two types $\theta$ and $k$ are common knowledge among bidders, they are unknown to the econometrician, which leads to a problem with multidimensional unobserved heterogeneity. ${ }^{12}$ The model primitives are the component value quantile functions $\left\{v_{k}(\cdot)\right\}_{k \in\{1, \ldots, K\}}$ and the proportion of each bidder type combination $\left\{p_{k \theta}\right\}_{k \in\{1, \ldots, K\}, \theta \in\left\{\theta_{H}, \theta_{L}\right\}}$.

We study identification of this model in several steps. Following An (2016), we assume that each bidder participates at least three independent auctions and the type is invariant. First, we can relabel the bidder's type as a combination of risk aversion level $\theta$ and the type of value distribution, i.e. $\tau=(k, \theta)$. There are $2 K$ types of bidders so $\tau=1, \ldots, 2 K$. Let $G_{k \theta}(\cdot)$ represent the bid distribution function generated from bidders whose value quantile function is $v_{k}(\cdot)$ and risk aversion level is $\theta$. Second, applying Theorem 2, we identify the component bid quantile functions $b_{\tau}(\cdot)$ up to permutation and the probability of type $\tau$. Obviously, identification of the value distribution functions relies on correctly assigning $\theta$ to the component bid quantile functions. Moreover, identification of $p_{k \theta}$ requires correctly assigning each $\tau$ to a pair $(\theta, k)$. However, identification up to permutation does not provide the correspondence between $\tau$ and $(k, \theta)$.

Now we describe how to apply our results to assigning $\theta$ to the list of "anonymous" bid quantile functions. It involves iterating the following two steps.

Step 1: We identify the lowest-ranked component bid distribution function and its corresponding value distribution function, say $F_{k_{1}}(\cdot)$. For any $k \in \mathcal{K}$, Appendix B shows that the bid quantile function of the type- $\left(k, \theta_{L}\right)$ bidders ranks higher than the type- $\left(k, \theta_{H}\right)$ bidders in our ordered tree that is constructed from $\left\{b_{\tau}(\cdot)\right\}_{\tau \in\{1, \ldots, 2 K\}}$. Therefore, the lowest ranked bid quantile function in our ordered tree correspond to the less risk averse bidders, i.e., $\theta=\theta_{H}$. Denote its distribution function as $G_{k_{1} \theta_{H}}(\cdot)$. This allows us to identify the inverse

\footnotetext{
${ }^{12}$ Identification of multidimensional unobserved heterogeneity is also studied by Aguirregabiria and Mira (2015) for static games of incomplete information and Xiao (2016) for dynamic games.
} 
bidding strategy, say $s_{k_{1} \theta_{H}}^{-1}(\cdot)$, and its corresponding value distribution function, say $F_{k_{1}}(\cdot)$, via Equation (4) with $\theta_{i}=\theta_{H}$.

Step 2: We identify the other component bid distribution function that corresponds to the same value distribution function $F_{k_{1}}(\cdot)$ but a different risk aversion level. In particular, Appendix B shows that

$$
\frac{d}{d b} \log F_{k_{1}}\left(s_{k_{1} \theta_{H}}^{-1}(b)\right)-\frac{d}{d b} \log F_{k_{1}}\left(s_{k_{1} \theta_{L}}^{-1}(b)\right)=\frac{\theta_{L}}{s_{k_{1} \theta_{L}}^{-1}(b)-b}-\frac{\theta_{H}}{s_{k_{1} \theta_{H}}^{-1}(b)-b}
$$

where $s_{k_{1} \theta_{L}}^{-1}(\underline{v})=\underline{v}$ and $s_{k_{1} \theta_{L}}^{-1}(\bar{b})=\bar{v}$. We can show that this two points boundary value problem identifies $s_{k_{1} \theta_{L}}^{-1}(\cdot)$, which make use of the following simple lemma.

Lemma 6. Let $h$ be a differentiable function on $[\underline{x}, \bar{x}]$ for which $(i) h(\bar{x})=0$, (ii) $h(x)>0$ implies that $h^{\prime}(x) \geq 0$ and (iii) $h(x)<0$ implies that $h^{\prime}(x) \leq 0$. Then $h(x)=0$ for all $x \in[\underline{x}, \bar{x}]$.

Lemma 7. The two points boundary value problem (5) has a unique solution $s_{k_{1} \theta_{L}}^{-1}(\cdot)$.

Finally, rewriting Equation (4) gives

$$
\frac{g_{k_{1} \theta_{L}}(b)}{G_{k_{1} \theta_{L}}(b)}=\sum_{\imath=1}^{I} \frac{g_{\imath}(b)}{G_{\imath}(b)}-\frac{\theta_{L}}{s_{k_{1} \theta_{L}}^{-1}(b)-b}
$$

which identifies $G_{k_{1} \theta_{L}}(\cdot)$ as follows:

$G_{k_{1} \theta_{L}}(b)=\exp \left\{-\int_{b}^{\bar{b}}\left[\sum_{\imath=1}^{I} \frac{g_{\imath}(x)}{G_{\imath}(x)}-\frac{\theta_{L}}{s_{k_{1} \theta_{L}}^{-1}(x)-x}\right] d x\right\}=\Pi_{\imath=1}^{I} G_{\imath}(b) \cdot \exp \left\{\int_{b}^{\bar{b}} \frac{\theta_{L}}{s_{k_{1} \theta_{L}}^{-1}(x)-x} d x\right\}$

which is derived from the value distribution function $F_{k_{1}}(\cdot)$ and risk aversion level $\theta_{L}$.

Dropping these two bid quantile functions from the list, $2(K-1)$ "anonymous" bid quantile functions remain. We again construct an ordered tree and identify the value quantile function that corresponds to the lowest ranked bid quantile function, as well as the bid quantile function of more risk averse bidders who have the same value distribution. We can 
repeat this process till each $\tau$ has been assigned a value of $\theta$ and a value of $k$.

In sum, with the same data requirements as in An (2016), the first-price auction model with unobserved asymmetry in both preferences and private values is identified.

\section{Empirical Application}

\subsection{Data}

In this section, we apply our results on empirical analysis of the United States Forest Service (USFS) timber auctions. See, e.g., Baldwin, Marshall, and Richard (1997), Haile (2001) and Haile and Tamer (2003). In particular, we analyze the sealed-bid auction data from 1982 to 1990, which are constructed from the data available on Philip A. Haile's website. Following Haile, Hong, and Shum (2003), we consider only scaled sales in Forest Service regions 1 and 5, which minimizes the significance of subcontracting/resale and thus common value. Moreover, we drop sales set aside for small businesses and salvage sales. In sum, we focus on the auctions that are most likely to satisfy the independent private value assumption. For simplicity, we focus on the 159 auctions with three bidders.

Table 1 provides summary statistics on a list of auction-specific observables: the winning bid, the size of the tract (in acres), the estimated volume of timber (in MBF), the appraisal value (per MBF), the estimated selling value (per MBF), the estimated harvesting cost (per $\mathrm{MBF}$ ), the estimated manufacturing cost (per MBF), the species concentration index (HHI) and the Forest Service region. ${ }^{13}$

Before implementing our method, we apply the Haile, Hong, and Shum (2003) method to homogenize the bids. Table 2 displays the regression results. Regression (3) includes all the control variables as well as year dummies. All of the estimated coefficients have the expected signs. We calculate the homogenized bids as the exponential of the differences between the

\footnotetext{
${ }^{13}$ We construct the variables following Liu and Luo (2016). All dollar values are nominal and all volume values are in thousand board-feet $(\mathrm{MBF})$ of timber.
} 
Table 1: Summary Statistics

\begin{tabular}{lllll}
\hline \hline Variable & Mean & Std. Dev. & Min & Max \\
\hline winning bid & 95688.19 & 148656.6 & 2848.18 & 1112966 \\
acres & 499.3522 & 791.237 & 4 & 7000 \\
vol_sum & 1776.107 & 2493.26 & 52 & 12900 \\
AppValue_avg & 29.48655 & 26.95233 & 0.5 & 162.6877 \\
SellValue_avg & 349.6786 & 68.21548 & 154.9083 & 532.5739 \\
LogCost_avg & 134.809 & 28.0862 & 64.81 & 259.44 \\
MfgCost_avg & 169.1068 & 33.87054 & 3.707538 & 248.6233 \\
HHI & 0.578792 & 0.244737 & 0.172212 & 1 \\
D5 & 0.465409 & 0.500378 & 0 & 1 \\
\hline
\end{tabular}

Note: D $5=1$ if the Forest Service region is $5,=0$ otherwise.

logarithm of the original total bids and the demeaned fitted values of regression (3).

Table 2: Regression Results

\begin{tabular}{lccc}
\hline VARIABLES & $(1)$ & $(2)$ & $(3)$ \\
\hline log_acres & & -0.0188 & -0.0330 \\
& & $(0.0252)$ & $(0.0236)$ \\
log_vol_sum & $1.014^{* * *}$ & $1.032^{* * *}$ & $1.058^{* * *}$ \\
& $(0.0213)$ & $(0.0289)$ & $(0.0293)$ \\
log_AppValue_avg & $0.595^{* * *}$ & $0.456^{* * *}$ & $0.458^{* * *}$ \\
& $(0.0343)$ & $(0.0470)$ & $(0.0446)$ \\
log_SellValue_avg & & $1.166^{* * *}$ & $1.242^{* * *}$ \\
& & $(0.201)$ & $(0.209)$ \\
log_LogCost_avg & & $-0.437^{* * *}$ & $-0.421^{* * *}$ \\
& & $(0.133)$ & $(0.131)$ \\
log_MfgCost_avg & & $-0.205^{* * *}$ & $-0.268^{* * *}$ \\
& & $(0.0641)$ & $(0.0645)$ \\
HHI & & -0.0973 & -0.0504 \\
& & $(0.103)$ & $(0.0977)$ \\
Observations & 477 & 477 & 477 \\
R-squared & 0.841 & 0.853 & 0.866 \\
Year FE & & & YES \\
\hline Robust standard errors in parentheses
\end{tabular}

Robust standard errors in parentheses

*** $\mathrm{p}<0.01, * * \mathrm{p}<0.05, * \mathrm{p}<0.1$ 


\subsection{Estimation Results}

In view of the small sample size, we estimate a parametric auction model when bidders are risk neutral. In particular, we propose the family of the two-sided power distributions, which is introduced into the statistic literature by Van Dorp and Kotz (2002). It has a location parameter $\theta$ and a shape parameter $\gamma$. The two-sided power distribution has two important advantages in the context of auctions. First, this family of distributions has similar flexibility as the Beta distribution. It includes the uniform distribution, the standard power distributions and the triangular distributions. Moreover, similar to the Beta distribution, one can also show that one can also approximate any continuous distribution function on $[0,1]$ arbitrarily close by a convex combination of two-sided power distribution functions (see Bornkamp and Ickstadt (2009)). Second, it is easy to solve the auction model when values are draws from the two-sided power distributions.

The two-sided power distribution function is given by

$$
F(x ; \theta, \gamma)=\left\{\begin{array}{ll}
\theta\left(\frac{x}{\theta}\right)^{\gamma} & \text { if } x \in[0, \theta] \\
1-(1-\theta)\left(\frac{1-x}{1-\theta}\right)^{\gamma} & \text { if } x \in(\theta, 1]
\end{array},\right.
$$

where the location parameter $\theta \in[0,1]$ and the shape parameter $\gamma>0$. Since the two-sided power distribution is supported on the range $[0,1]$, we alter the location and scale of the distribution by introducing a linear transformation using an additional location parameter $\kappa_{0}$ and a scale parameter $\kappa_{1}$. In particular, we assume that the values satisfy $v_{i}=\kappa_{0}+$ $\kappa_{1} x_{i}$, where $i \in\{1,2,3\}$, and $x_{i}$ 's are i.i.d. draws from the two-sided power distribution conditioning on the auction-specific state $(\gamma, \theta)$.

For simplicity, assume that each of the state parameters $(\gamma, \theta)$ has two possible values, i.e., $\gamma \in\left\{\gamma_{L}, \gamma_{H}\right\}$ and $\theta \in\left\{\theta_{L}, \theta_{H}\right\}$, where $\gamma_{L}<\gamma_{H}$ and $\theta_{L}<\theta_{H}$. Two-dimensional unobserved heterogeneity arises because the analyst does not observe $(\gamma, \theta)$. The primitives are $\left(\kappa_{0}, \kappa_{1}\right)$, the location and shape parameters $\left\{\gamma_{L}, \gamma_{H}, \theta_{L}, \theta_{H}\right\}$ and the distribution of the state 
$\left\{p_{L L}, p_{L H}, p_{H L}, p_{H H}\right\}$, where the two subscripts represent the levels of $\gamma$ and $\theta$, respectively.

We estimate the model via maximum likelihood. ${ }^{14}$ See, e.g., Donald and Paarsch (1993), Hirano and Porter (2003) and Chernozhukov and Hong (2004). All dollar values are reported in thousands. $\kappa_{0}$ is estimated to be the minimum homogenized bid, i.e. 2.63. The estimated scale parameter is $\widehat{\kappa}_{1}=187.59$. The estimated location and shape parameters are $\widehat{\gamma}_{L}=$ 1.00, $\widehat{\gamma}_{H}=5.34, \widehat{\theta}_{L}=0.0896, \widehat{\theta}_{H}=0.1610$. The mixing weights are $\widehat{p}_{L L}=0.2612, \widehat{p}_{L H}=$ $0.2365, \widehat{p}_{H L}=0.2459, \widehat{p}_{H H}=0.2564$. Note that the two-sided power distribution becomes a uniform distribution when $\gamma=1$. Thus, assuming that our model is correctly specified, our results implies that the bids that we observe are mostly likely from a mixture of a uniform distribution, and two two-sided power distributions with parameters $\left(\widehat{\gamma}_{H}, \widehat{\theta}_{L}\right)$ and $\left(\widehat{\gamma}_{H}, \widehat{\theta}_{H}\right)$, respectively. The mixing weights are $49.77 \%, 24.59 \%$ and $25.64 \%$, respectively. Figure 4 shows the three component value distribution functions.

Figure 4: Estimated Component Value Distributions

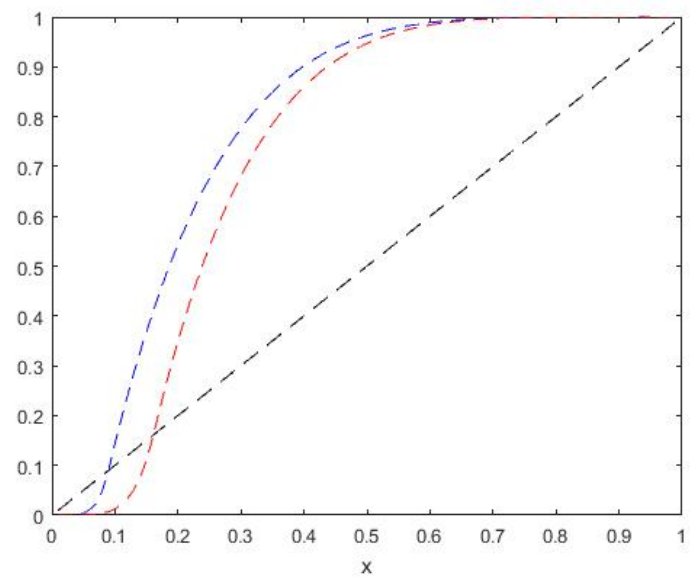

\section{Conclusion}

In this paper, we introduce the concept of restricted stochastic dominance in auctions. We show that the same relationship passes from the value quantile functions to the bid quantile

\footnotetext{
${ }^{14}$ We use the Nelder-Mead Simplex Method for maximizing the objective function. Our estimation converges to the same solution for all attempted starting values.
} 
functions. Relying on these results, we define a RSD ordering for a set of distinct quantile functions and show that this ordering is preserved by the mapping from value quantile function to bid quantile function in symmetric first-price auctions. This property is used to generalize Hu, McAdams, and Shum (2013) by allowing nonseparable unobserved heterogeneity under an RSD condition. This condition is more general than the first-order stochastic dominance condition and it allows all analytic functions as well as some non-analytic ones. Therefore, our results further expand the breadth of applicability of their misclassification approach. Finally, we apply our results to identification of various auction models with unobserved heterogeneity.

While we focus on auction models in this paper, the RSD ordering can be useful in other applications of measurement error models. See Hu (2015) for a recent survey on applications in empirical industrial organization and labor economics. Moreover, although we study only discrete unobserved heterogeneity in this paper, the RSD ordering can also be applied in the continuous case. See, e.g., Hu and Schennach (2008). We leave this for future research. 


\section{Appendices}

\section{A Proofs}

\section{A.1 Proof of Lemma 1}

Proof. Consider an arbitrary $\epsilon>0$. First, consider $v_{1}(\cdot)$ and $v_{2}(\cdot)$. There exists $x_{12} \in$ $\left[\alpha_{\dagger}, \alpha_{\dagger}+\epsilon\right)$ such that $\forall \alpha \in\left[0, x_{12}\right], v_{1}(\alpha) \leq v_{2}(\alpha), \& \exists \alpha_{12} \in\left(\alpha_{\dagger}, x_{12}\right], v_{1}\left(\alpha_{12}\right)<v_{2}\left(\alpha_{12}\right)$. Second, consider $v_{2}(\cdot)$ and $v_{3}(\cdot)$. There exists $x_{23} \in\left[\alpha_{\dagger}, \alpha_{12}\right)$ such that $\forall \alpha \in\left[0, x_{23}\right], v_{2}(\alpha) \leq$ $v_{3}(\alpha), \& \exists \alpha_{23} \in\left(\alpha_{\dagger}, x_{23}\right], v_{2}\left(\alpha_{23}\right)<v_{3}\left(\alpha_{23}\right)$. Note that $0 \leq \alpha_{\dagger}<\alpha_{23} \leq x_{23}<\alpha_{12} \leq x_{12}<$ $\alpha_{\dagger}+\epsilon$. In sum, $\alpha_{23} \in\left[0, x_{23}\right] \subset\left[0, x_{12}\right]$ and $v_{1}\left(\alpha_{23}\right) \leq v_{2}\left(\alpha_{23}\right)<v_{3}\left(\alpha_{23}\right)$, where the first inequality follows $\forall \alpha \in\left[0, x_{12}\right], v_{1}(\alpha) \leq v_{2}(\alpha)$. Moreover, $\forall \alpha \in\left[0, x_{23}\right], v_{1}(\alpha) \leq v_{2}(\alpha) \leq$ $v_{3}(\alpha)$. Therefore, $v_{1}(\cdot) \underset{\alpha_{\dagger}}{\prec} v_{3}(\cdot)$.

\section{A.2 Proof of Lemma 2}

Proof. Consider an arbitrary $\epsilon>0$. We consider three exhaustive and exclusive cases: (1) If $\alpha_{\dagger}=\alpha_{\dagger \dagger}$, we obtain $v_{1}(\cdot) \underset{\alpha_{\dagger}}{\prec} v_{3}(\cdot)$ because Lemma 1 applies. (2) If $\alpha_{\dagger}<\alpha_{\dagger \dagger}$, $v_{2}(\cdot) \underset{\alpha_{\dagger \dagger}}{\prec} v_{3}(\cdot)$ implies that there exists $x_{23} \in\left[\alpha_{\dagger \dagger}, \alpha_{\dagger \dagger}+\epsilon\right)$ such that $\forall \alpha \in\left[0, x_{23}\right], v_{2}(\alpha) \leq$ $v_{3}(\alpha), \& \exists \alpha_{23} \in\left(\alpha_{\dagger \dagger}, x_{23}\right], v_{2}\left(\alpha_{23}\right)<v_{3}\left(\alpha_{23}\right)$. Moreover, $v_{1}(\cdot) \underset{\alpha_{\dagger}}{\prec} v_{2}(\cdot)$ implies that there exists $x_{12} \in\left[\alpha_{\dagger}, \min \left\{\alpha_{23}, \alpha_{\dagger}+\epsilon\right\}\right)$ such that $\forall \alpha \in\left[0, x_{12}\right], v_{1}(\alpha) \leq v_{2}(\alpha), \& \exists \alpha_{12} \in\left(\alpha_{\dagger}, x_{12}\right], v_{1}\left(\alpha_{12}\right)<$ $v_{2}\left(\alpha_{12}\right)$. Note that $0 \leq \alpha_{\dagger}<\alpha_{12} \leq x_{12}<\alpha_{23} \leq x_{23}<\alpha_{\dagger \dagger}+\epsilon$. In sum, $\alpha_{12} \in\left[0, x_{12}\right] \subset\left[0, x_{23}\right]$ and $v_{1}\left(\alpha_{12}\right)<v_{2}\left(\alpha_{12}\right) \leq v_{3}\left(\alpha_{12}\right)$, where the second inequality follows $\forall \alpha \in\left[0, x_{23}\right], v_{2}(\alpha) \leq$ $v_{3}(\alpha)$. Moreover, $\forall \alpha \in\left[0, x_{12}\right], v_{1}(\alpha) \leq v_{2}(\alpha) \leq v_{3}(\alpha)$. Therefore, $v_{1}(\cdot) \underset{\alpha_{\dagger}}{\prec} v_{3}(\cdot)$. (3) If $\alpha_{\dagger}>\alpha_{\dagger \dagger}$, we obtain $v_{1}(\cdot) \underset{\alpha_{\dagger \dagger}}{\prec} v_{3}(\cdot)$ following similar lines as in (2).

\section{A.3 Proof of Lemma 3}

Proof. We prove by contradiction. Suppose $b_{1}\left(\alpha_{R}\right)>b_{2}\left(\alpha_{R}\right)$ for some $\alpha_{R} \in[0, x]$. Let $\alpha_{L} \equiv \inf \left\{x^{\prime} \in\left(0, \alpha_{R}\right] \mid b_{1}(\alpha)>b_{2}(\alpha), \forall \alpha \in\left(x^{\prime}, \alpha_{R}\right]\right\}$. From the continuity of $b_{k}(\cdot) \mathrm{s}$, we have $b_{1}\left(\alpha_{L}\right)=b_{2}\left(\alpha_{L}\right)$. On the other hand, since $b_{1}(\alpha)>b_{2}(\alpha), \forall \alpha \in\left(\alpha_{L}, \alpha_{R}\right]$, we have 
$b_{1}^{\prime}(\alpha)<b_{2}^{\prime}(\alpha), \forall \alpha \in\left(\alpha_{L}, \alpha_{R}\right]$. In fact, since $v_{1}(\alpha) \leq v_{2}(\alpha), \forall \alpha \in[0, x]$, we have $v_{1}(\alpha)-$ $b_{1}(\alpha)<v_{2}(\alpha)-b_{2}(\alpha), \forall \alpha \in\left(\alpha_{L}, \alpha_{R}\right]$. Thus, monotonicity of $\lambda(\cdot)$ implies that

$$
\frac{I-1}{\alpha} \lambda\left(v_{1}(\alpha)-b_{1}(\alpha)\right)<\frac{I-1}{\alpha} \lambda\left(v_{2}(\alpha)-b_{2}(\alpha)\right)
$$

which implies that $b_{1}^{\prime}(\alpha)<b_{2}^{\prime}(\alpha), \forall \alpha \in\left(\alpha_{L}, \alpha_{R}\right]$ due to Equation (2). Therefore, $b_{1}\left(\alpha_{R}\right)=$ $b_{1}\left(\alpha_{L}\right)+\int_{\alpha_{L}}^{\alpha_{R}} b_{1}^{\prime}(x) d x<b_{2}\left(\alpha_{L}\right)+\int_{\alpha_{L}}^{\alpha_{R}} b_{2}^{\prime}(x) d x=b_{2}\left(\alpha_{R}\right)$, a contradiction.

\section{A.4 Proof of Lemma 4}

Proof. Let $\alpha_{L}=\sup \left\{x^{\prime} \in\left[0, \alpha_{*}\right) \mid v_{1}(\alpha)=v_{2}(\alpha), \forall \alpha \in\left[0, x^{\prime}\right]\right\}$. By continuity of $v_{k}(\cdot), \alpha_{L}<\alpha_{*}$ and $v_{1}\left(\alpha_{L}\right)=v_{2}\left(\alpha_{L}\right)$. Lemma 3 implies that $b_{1}\left(\alpha_{L}\right)=b_{2}\left(\alpha_{L}\right)$ as well. By definition of $\alpha_{L}$, for any $\epsilon>0$, there exists a probability $\alpha \in\left(\alpha_{L}, \alpha_{L}+\epsilon\right)$ such that $v_{1}(\alpha)<v_{2}(\alpha)$.

Consider a probability $\alpha \in\left(\alpha_{L}, x\right]$. Equation (2) implies that

$$
\begin{aligned}
\alpha\left[b_{2}^{\prime}(\alpha)-b_{1}^{\prime}(\alpha)\right] & =(I-1)\left[\lambda\left(v_{2}(\alpha)-b_{2}(\alpha)\right)-\lambda\left(v_{1}(\alpha)-b_{1}(\alpha)\right)\right] \\
& =(I-1) \lambda^{\prime}(\widetilde{R(\alpha)})\left[\left(v_{2}(\alpha)-v_{1}(\alpha)\right)-\left(b_{2}(\alpha)-b_{1}(\alpha)\right)\right],
\end{aligned}
$$

where the second equation follows the mean value theorem and $\widetilde{R(\alpha)}$ is a value between $v_{1}(\alpha)-b_{1}(\alpha)$ and $v_{2}(\alpha)-b_{2}(\alpha)$. Denote $y(\alpha)=b_{2}(\alpha)-b_{1}(\alpha), q(\alpha)=-(I-1) \lambda^{\prime}(\widetilde{R(\alpha)}) / \alpha, p(\alpha)=$ $(I-1)\left(v_{2}(\alpha)-v_{1}(\alpha)\right) \lambda^{\prime}(\widetilde{R(\alpha)}) / \alpha$. We obtain $y^{\prime}(\alpha)=q(\alpha) y(\alpha)+p(\alpha)$, whose solutions are of the form

$$
y(\alpha)=c e^{Q(\alpha)}+e^{Q(\alpha)} \int_{\alpha_{L}}^{\alpha} e^{-Q(x)} p(x) d x,
$$

where $Q(\alpha)=\int_{\alpha_{L}}^{\alpha} q(x) d x$ and $c$ is a constant. The boundary condition $y\left(\alpha_{L}\right)=b_{2}\left(\alpha_{L}\right)-$ $b_{1}\left(\alpha_{L}\right)=0$ implies that $c=0$. Since $\lambda^{\prime}(\cdot)=\left(U(\cdot) / U^{\prime}(\cdot)\right)^{\prime}=1-U(\cdot) U^{\prime \prime}(\cdot) /\left(U^{\prime}(\cdot)\right)^{2} \geq$ $1, v_{2}(\alpha)-v_{1}(\alpha) \geq 0, \forall \alpha \in\left(\alpha_{L}, \alpha\right]$ and $v_{2}\left(\alpha^{\prime}\right)-v_{1}\left(\alpha^{\prime}\right)>0$ for some $\alpha^{\prime} \in\left(\alpha_{L}, \alpha\right)$, we have $p(\alpha) \geq 0, \forall \alpha \in\left(\alpha_{L}, \alpha\right]$ and $p\left(\alpha^{\prime}\right)>0$ for some $\alpha^{\prime} \in\left(\alpha_{L}, \alpha\right)$. Therefore, $y(\alpha)=$ $\int_{\alpha_{L}}^{\alpha} e^{Q(\alpha)-Q(x)} p(x) d x>0$. In other words, $b_{2}(\alpha)>b_{1}(\alpha), \forall \alpha \in\left(\alpha_{L}, x\right]$. 


\section{A.5 Proof of Equation 3}

Proof. For simplicity, consider homogeneous auctions. First, bidders' valuation quantile function satisfies the FOC

$$
v(\alpha)=b_{I}(\alpha)+\frac{1}{I-1} \alpha b_{I}^{\prime}(\alpha) .
$$

Let $y(\alpha)=b(\alpha), q(\alpha)=-(I-1) / \alpha, p(\alpha)=(I-1) v(\alpha) / \alpha$. We obtain $y^{\prime}(\alpha)=q(\alpha) y(\alpha)+$ $p(\alpha)$, whose solutions are of the form

$$
y(\alpha)=c e^{Q(\alpha)}+e^{Q(\alpha)} \int_{0}^{\alpha} e^{-Q(x)} p(x) d x,
$$

where $Q(\alpha)=\int_{0}^{\alpha} q(x) d x$ and $c$ is a constant. Note that $Q(\alpha)-Q(x)=\int_{x}^{\alpha} q(z) d z=$ $\ln (x / \alpha)^{I-1}$. The boundary condition $y(0)=\underline{v}$ implies that $c=0$. Rearranging terms leads to an explicit mapping from valuation quantile function to bid quantile function

$$
b_{I}(\alpha)=(I-1) \alpha^{1-I} \int_{0}^{\alpha} v(x) x^{I-2} d x .
$$

See also Gimenes and Guerre (2016) and Liu and Luo (2016). Therefore, the two bid quantile functions under different competition levels satisfy the following condition

$$
b_{I_{2}}(\alpha)=\left(I_{2}-1\right) \alpha^{1-I_{2}} \int_{0}^{\alpha}\left[b_{I_{1}}(x)+\frac{1}{I_{1}-1} x b_{I_{1}}^{\prime}(x)\right] x^{I_{2}-2} d x .
$$

Integration by parts gives

$$
b_{I_{2}}(\alpha)=\frac{I_{2}-1}{I_{1}-1}\left[b_{I_{1}}(\alpha)+\left(I_{1}-I_{2}\right) \alpha^{1-I_{2}} \int_{0}^{\alpha} b_{I_{1}}(x) x^{I_{2}-2} d x\right] .
$$

Moreover, it is also known that $b_{I_{1}}(\cdot)<b_{I_{2}}(\cdot)$ on $(0,1]$ if $I_{1}<I_{2}$, and $b_{I_{1}}(0)=b_{I_{2}}(0)=\underline{v}$. See Guerre, Perrigne, and Vuong (2009). 


\section{A.6 Proof of Theorem 3}

Proof. Note that $L_{D_{j}, D_{i}}=L_{D_{j} \mid K} \times D_{K} \times L_{D_{i} \mid K}^{T}$, where $D_{K}=\operatorname{diag}\left\{\operatorname{Pr}\{K=k\}_{k \in\{1, \ldots, K\}}\right\}$ has rank $K$. We only need to show that both $L_{D_{j} \mid K}$ and $L_{D_{i} \mid K}$ have rank $K$.

For an arbitrary list of cutoff points $x_{1}<\ldots<x_{\imath}$,

$$
L_{D_{j} \mid K}=\left(\begin{array}{llll}
F_{1}\left(x_{1}\right)-0 & F_{2}\left(x_{1}\right)-0 & \ldots & F_{K}\left(x_{1}\right)-0 \\
F_{1}\left(x_{2}\right)-F_{1}\left(x_{1}\right) & F_{2}\left(x_{2}\right)-F_{2}\left(x_{1}\right) & \ldots & F_{K}\left(x_{2}\right)-F_{K}\left(x_{1}\right) \\
\ldots & \ldots & \ldots & \ldots \\
F_{1}\left(x_{\imath}\right)-F_{1}\left(x_{\imath-1}\right) & F_{2}\left(x_{\imath}\right)-F_{2}\left(x_{\imath-1}\right) & \ldots & F_{K}\left(x_{\imath}\right)-F_{K}\left(x_{\imath-1}\right) \\
1-F_{1}\left(x_{\imath}\right) & 1-F_{2}\left(x_{\imath}\right) & \ldots & 1-F_{K}\left(x_{\imath}\right)
\end{array}\right)
$$

By adding every row to all its later ones and then reordering rows, we obtain the following matrix which has the same rank as $L_{D_{j} \mid K}$ :

$$
A_{\imath}=\left(\begin{array}{cccc}
1 & 1 & \ldots & 1 \\
F_{1}\left(x_{1}\right) & F_{2}\left(x_{1}\right) & \ldots & F_{K}\left(x_{1}\right) \\
F_{1}\left(x_{2}\right) & F_{2}\left(x_{2}\right) & \ldots & F_{K}\left(x_{2}\right) \\
\ldots & \ldots & \ldots & \ldots \\
F_{1}\left(x_{\imath}\right) & F_{2}\left(x_{\imath}\right) & \ldots & F_{K}\left(x_{\imath}\right)
\end{array}\right)
$$

For notational convenience, we look for a list of cutoff points such that $A_{\iota}$ has rank $K$.

The proof has two steps. In the first step, we show there exists a list of cutoff points such that $A_{\imath}$ is of rank $K$ by adding well-chosen points to an arbitrary list of cutoff points. Take an arbitrary list of cutoff points $x_{1}<x_{2}<\ldots<x_{\imath}$, where $\imath \geq K-1$. If $A_{\imath}$ is of rank $K$, we move directly to the second step of the proof. If $A_{\imath}$ is not of rank $K$, we can find a nonzero vector $\beta \in R^{K}$ such that $A_{\imath} \beta=0$. Since the distributions are linearly independent, we can 
find $x_{\imath+1}$ such that $\sum_{k=1}^{K} F_{k}\left(x_{\imath+1}\right) \beta_{k} \neq 0$. Let

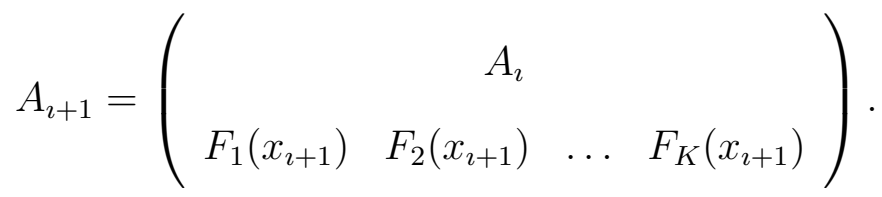

We now show that $A_{\imath+1}$ has a larger rank than $A_{\imath}$. If not, they are of the same rank, which implies that the new row is a linear combination of the original rows. That is, $F_{k}\left(x_{\imath+1}\right)=\sum_{\ell=0}^{\imath} \gamma_{\ell} F_{k}\left(x_{\ell}\right)$ for some $\left(\gamma_{0}, \ldots, \gamma_{\imath}\right)^{\prime}$, where $F_{k}\left(x_{0}\right)=1$. Since $\sum_{k=1}^{K} F_{k}\left(x_{\imath+1}\right) \beta_{k} \neq$ 0 , we have $\sum_{k=1}^{K}\left[\sum_{\ell=0}^{\imath} \gamma_{\ell} F_{k}\left(x_{\ell}\right)\right] \beta_{k} \neq 0$. On the other hand, $A_{\imath} \beta=0$ implies that $0=$ $\sum_{\ell=0}^{\imath} \gamma_{\ell}\left[\sum_{k=1}^{K} F_{k}\left(x_{\ell}\right) \beta_{k}\right]=\sum_{k=1}^{K}\left[\sum_{\ell=0}^{\imath} \gamma_{\ell} F_{k}\left(x_{\ell}\right)\right] \beta_{k}$, a contradiction.

We can continue adding new rows in this manner till the rank increases to $K$. Without loss of generality, denote the cutoff points as $x_{1}<x_{2}<\ldots<x_{\imath}$, where $\imath \geq K-1$.

In the second step, based on the list $\left\{x_{1}, \ldots, x_{\imath}\right\}$, we can find exactly $K-1$ cutoff points $\left\{x_{1}, \ldots, x_{K-1}\right\}$ such that $A_{K}$ is of rank $K$ by applying Lemma 2 in Xiao (2015).

\section{A.7 Proof of Theorem 4}

Proof. Suppose $v_{k}(\cdot)$ and $v_{k^{\prime}}(\cdot)$ are analytic and Assumption 4 is not true. Then, for any $x \in(0,1]$, we can find a switch of sign for the function $v_{k}(\cdot)-v_{k^{\prime}}(\cdot)$ in $[0, x]$. By the intermediate value theorem, there exists an $\alpha_{(1)}$ in $(0,1]$ such that $v_{k}\left(\alpha_{(1)}\right)=v_{k^{\prime}}\left(\alpha_{(1)}\right)$. For the same reason, we can find an $\alpha_{(2)}$ in $\left(0, \min \left\{1 / 2, \alpha_{(1)}\right\}\right]$ such that $v_{k}\left(\alpha_{(2)}\right)=v_{k^{\prime}}\left(\alpha_{(2)}\right)$. Repeating this procedure yields a converging sequence $\left\{\alpha_{(m)}\right\}_{m=1,2, \ldots}$, where $\alpha_{(m)} \in\left(0, \min \left\{1 / 2^{m-1}, \alpha_{(m-1)}\right]\right.$, such that $v_{k}\left(\alpha_{(m)}\right)=v_{k^{\prime}}\left(\alpha_{(m)}\right)$, and $\lim _{m \rightarrow \infty} \alpha_{(m)}=0$. Note that $v_{k}(0)-v_{k^{\prime}}(0)=0$. Therefore, the difference must be 0 in $(0,1]$ by the principle of permanence, meaning that $v_{k}(\alpha)=v_{k^{\prime}}(\alpha), \forall \alpha \in[0,1]$, a contradiction.

\section{A.8 Proof of Lemma 5}

Proof. Consider two different Beta distributions defined by $\left(\alpha_{1}, \beta_{1}\right)$ and $\left(\alpha_{2}, \beta_{2}\right)$, respectively. Note that if $f_{1} \leq f_{2}, \forall a \in[0, x]$, then $F_{1} \leq F_{2}, \forall a \in[0, x]$. Thus, we only need to show that 
there exists a point $x$ such that the difference between the two pdfs has no change of sign on $(0, x)$. We proof by contradiction. Suppose that for any $x$, we can find a change of sign on $(0, x)$ and thus a point in $(0, x)$ such that the two pdf's are the same. Take a point $x$ where the two pdf's are the same, i.e. $x^{\alpha_{1}-1}(1-x)^{\beta_{1}-1} / B_{1}=x^{\alpha_{2}-1}(1-x)^{\beta_{2}-1} / B_{2}$, where $B_{t}=\int_{0}^{1} x^{\alpha_{t}-1}(1-x)^{\beta_{t}-1} d x$. Taking the logarithm gives

$$
T(x) \equiv\left(\alpha_{2}-\alpha_{1}\right) \log x+\left(\beta_{2}-\beta_{1}\right) \log (1-x)-\log \left(B_{2} / B_{1}\right)=0
$$

Note that for any natural number $M$, we can find a point $x_{M}$ such that $x_{M} \in\left(0,1 / 2^{M}\right)$ and $T\left(x_{M}\right)=0$. Consider two cases: (1) if $\alpha_{2}=\alpha_{1}$ or $\beta_{2}=\beta_{1}, T(\cdot)$ can only have one zero point, a contradiction. (2) if $\alpha_{2} \neq \alpha_{1}$ and $\beta_{2} \neq \beta_{1}$. When $M \rightarrow+\infty$, we have $x_{M} \rightarrow 0, \log x_{M} \rightarrow-\infty, \log \left(1-x_{M}\right) \rightarrow 0$. Therefore, for an arbitrary $\epsilon>0,\left\|T\left(x_{M}\right)\right\|>\epsilon$ when $M$ is large enough, a contradiction.

\section{A.9 Proof of Theorem 7}

Proof. For a given $I=I_{1}, I_{2}$, we need to show that the same ordering passes from the value quantile functions to the bid quantile functions under Assumption 6. To this end, we focus on homogeneous auctions and show that $v^{\prime}(0)=\frac{I}{I-1} b^{\prime}(0)$.

Equation (1) implies that

$$
v^{\prime}(\alpha)=b^{\prime}(\alpha)+\frac{1}{I-1} \times \lambda^{-1 \prime}\left(\frac{1}{I-1} \alpha b^{\prime}(\alpha)\right) \times\left[\alpha b^{\prime \prime}(\alpha)+b^{\prime}(\alpha)\right] .
$$

Therefore,

$$
v^{\prime}(0)=\left[1+\frac{1}{I-1} \lambda^{-1 \prime}(0)\right] b^{\prime}(0)=\frac{I}{I-1} b^{\prime}(0)
$$

In fact, note that $\lambda(\cdot)=U(\cdot) / U^{\prime}(\cdot)$ and we assume $U(0)=0, U^{\prime}(\cdot)>0, U^{\prime \prime}(\cdot) \leq 0$. Thus, $\lambda(0)=0$. Note that $\lambda^{\prime}(\alpha)=\frac{\left(U^{\prime}(\cdot)\right)^{2}-U(\cdot) U^{\prime \prime}(\cdot)}{\left(U^{\prime}(\cdot)\right)^{2}}$. Thus, $\lambda^{\prime}(0)=\left(U^{\prime}(0)\right)^{2} /\left(U^{\prime}(0)\right)^{2}=1$. In sum, $\lambda^{-1 \prime}(0)=1 / \lambda^{\prime}(0)=1$ 


\section{A.10 Proof of Lemma 6}

Proof. We proceed by contradiction. Without loss of generality, suppose that there exists a point $x_{*} \in[\underline{x}, \bar{x})$ such that $h\left(x_{*}\right)>0$. Let $x_{L} \equiv \inf \left\{x \in\left(x_{*}, \bar{x}\right] \mid h(x)=0\right\}$. From the continuity of $h(\cdot)$, we have $h\left(x_{L}\right)=0$. Moreover, $h(x)>0, \forall x \in\left(x_{*}, x_{L}\right)$. Otherwise we have a contradiction of the definition of $x_{L}$ in either of the two exhaustive cases: (1) $h\left(x_{1}\right)=0$ for some $x_{1} \in\left(x_{*}, x_{L}\right) ;(2) h\left(x_{2}\right)<0$ for some $x_{2} \in\left(x_{*}, x_{L}\right)$. In this case, by the intermediate value theorem, there exists another $x_{3} \in\left(x_{*}, x_{2}\right)$ such that $h\left(x_{3}\right)=0$.

Since $h(x)>0, \forall x \in\left(x_{*}, x_{L}\right)$, Condition (ii) implies that $h^{\prime}(x) \geq 0, \forall x \in\left(x_{*}, x_{L}\right)$. However, by the mean value theorem, there exists a point $x \in\left(x_{*}, x_{L}\right)$ such that $h^{\prime}(x)=$ $\frac{h\left(x_{L}\right)-h\left(x_{*}\right)}{x_{L}-x_{*}}<0$, which contradicts the statement that $h^{\prime}(x) \geq 0, \forall x \in\left(x_{*}, x_{L}\right)$.

\section{Proof of Lemma 7}

Proof. Consider two solutions $\xi(\cdot)$ and $\widetilde{\xi}(\cdot)$. Denote $h(x)=\log F_{k_{1}}(\widetilde{\xi}(\cdot))-\log F_{k_{1}}(\xi(\cdot))$. Thus, $h(\bar{b})=0$ and $\frac{d}{d b} \log F_{k_{1}}(\xi(b))+\frac{\theta_{L}}{\xi(b)-b}=\frac{d}{d b} \log F_{k_{1}}(\widetilde{\xi}(b))+\frac{\theta_{L}}{\tilde{\xi}(b)-b}$, which implies that

$$
\frac{d}{d b} \log F_{k_{1}}(\widetilde{\xi}(b)) / F_{k_{1}}(\xi(b))=\frac{\theta_{L}(\widetilde{\xi}(b)-\xi(b))}{(\xi(b)-b)(\widetilde{\xi}(b)-b)} .
$$

Thus, (i) if $h(b)>0$, then $\widetilde{\xi}(b)>\xi(b)$ and also $h^{\prime}(b)=\frac{d}{d b} \log F_{k_{1}}(\widetilde{\xi}(b)) / F_{k_{1}}(\xi(b))>0$ and (ii) if $h(b)<0$, then $\widetilde{\xi}(b)<\xi(b)$ and also $h^{\prime}(b)=\frac{d}{d b} \log F_{k_{1}}(\widetilde{\xi}(b)) / F_{k_{1}}(\xi(b))<0$. Lemma 6 implies that $h(b)=0$ for all $b \in[\underline{b}, \bar{b}]$. In other words, $\widetilde{\xi}(\cdot)=\xi(\cdot)$.

\section{B Restricted Stochastic Dominance in Asymmetric Auctions}

Asymmetry among bidders can arise from either different valuation distributions $F_{i}(\cdot)$ and/or different utility functions $U_{i}(\cdot)$. In an independent private value first-price auction, bidder $i$ maximizes the payoff

$$
U_{i}(v-b) \cdot \prod_{j \neq i} F_{j}\left(s_{j}^{-1}(b)\right),
$$


where $\prod_{j \neq i} F_{j}\left(s_{j}^{-1}(b)\right)$ is the probability of winning. After some algebra, the FOC can be rewritten as

$$
\frac{1}{\lambda_{i}(v-b)}=\sum_{j \neq i} \frac{f_{j}\left(s_{j}^{-1}(b)\right)}{F_{j}\left(s_{j}^{-1}(b)\right)} \frac{1}{s_{j}^{\prime}\left(s_{j}^{-1}(b)\right)}
$$

For notation simplicity, we first consider the RSD ordering in auctions with asymmetry in private values and then in auctions with asymmetry in preferences.

\section{Asymmetry in Private Values}

Consider the asymmetric IPV model with risk neutral bidders. Assume that the $I$ bidders have potentially different value distributions $F_{k}(\cdot)$ with support $[\underline{v}, \bar{v}]$, where $k=1, \ldots, I$. Lebrun (1999) and Maskin and Riley (2000) show that the equilibrium is characterized by

$$
\frac{d}{d b} \xi_{k}(b)=\frac{F_{k}\left(\xi_{k}(b)\right)}{(I-1) f_{k}\left(\xi_{k}(b)\right)}\left\{\sum_{\ell=1}^{I} \frac{1}{\xi_{\ell}(b)-b}-\frac{I-1}{\xi_{k}(b)-b}\right\},
$$

along with the boundary conditions: $\xi_{k}(\bar{b})=\bar{v}$ and $\xi_{k}(\underline{v})=\underline{v}$, where $\xi_{k}(\cdot) \equiv s_{k}^{-1}(\cdot)$ is the inverse bidding strategy. We remark the theory predicts that bidders' bids share the same support. In contrast to symmetric auctions, we focus on the distribution functions instead of the quantile functions. Moreover, we treat the graph of $F_{k}(\cdot)$ as directioned while letting $(\bar{v}, 1)$ and $(\underline{v}, 0)$ be our starting point and ending point, respectively.

Suppose $F_{i}(\cdot) \geq F_{j}(\cdot)$ in $[z, \bar{v}]$, where $i \neq j \in\{1, \ldots, I\}$. We now show that the same RSD relation passes to the bid distributions, i.e. $G_{i}(\cdot) \geq G_{j}(\cdot)$ on $\left[s_{i}(z), \bar{b}\right]$. In general, no closed-form solution exists, which excludes direct comparisons.

First, note that $\frac{d}{d b} \log F_{k}\left(\xi_{k}(b)\right)=\frac{f_{k}\left(\xi_{k}(b)\right)}{F_{k}\left(\xi_{k}(b)\right)}\left[\frac{d}{d b} \xi_{k}(b)\right]$. The FOC becomes

$$
\frac{d}{d b} \log F_{k}\left(\xi_{k}(b)\right)=\frac{1}{I-1} \sum_{\ell=1}^{I} \frac{1}{\xi_{\ell}(b)-b}-\frac{1}{\xi_{k}(b)-b}
$$


which implies that

$$
\frac{d}{d b} \log F_{j}\left(\xi_{j}(b)\right)-\frac{d}{d b} \log F_{i}\left(\xi_{i}(b)\right)=\frac{1}{\xi_{i}(b)-b}-\frac{1}{\xi_{j}(b)-b} .
$$

Plugging in $b=s_{i}(v)$ and changing the differentiation to $v$ gives

$$
\frac{d}{d v} \log F_{j}\left(\xi_{j}\left(s_{i}(v)\right)\right)=\frac{d}{d v} \log F_{i}(v)+s_{i}^{\prime}(v)\left[\frac{1}{v-s_{i}(v)}-\frac{1}{\xi_{j}\left(s_{i}(v)\right)-s_{i}(v)}\right] .
$$

Second, $F_{j}\left(F_{j}^{-1}\left(F_{i}(v)\right)\right)=F_{i}(v)$ implies that

$$
\frac{d}{d v} \log F_{j}\left(F_{j}^{-1}\left(F_{i}(v)\right)\right)=\frac{d}{d v} \log F_{i}(v)
$$

Note that: 1$), \log F_{j}\left(F_{j}^{-1}\left(F_{i}(\bar{v})\right)\right)=\log F_{i}(\bar{v})=0$ and $\log F_{j}\left(\xi_{j}\left(s_{i}(\bar{v})\right)\right)=0$; 2), If $\log F_{j}\left(\xi_{j}\left(s_{i}(v)\right)\right)=\log F_{j}\left(F_{j}^{-1}\left(F_{i}(v)\right)\right)$ in $\left[v_{\dagger}, \bar{v}\right]$, we have $\xi_{j}\left(s_{i}(v)\right)=F_{j}^{-1}\left(F_{i}(v)\right) \geq v$ because $F_{i}(v) \geq F_{j}(v)$. Thus, $\left[\frac{1}{v-s_{i}(v)}-\frac{1}{\xi_{j}\left(s_{i}(v)\right)-s_{i}(v)}\right]=\frac{\xi_{j}\left(s_{i}(v)\right)-v}{\left(v-s_{i}(v)\right)\left(\xi_{j}\left(s_{i}(v)\right)-s_{i}(v)\right)} \geq 0$. Equations (6) and (7) imply that $\frac{d}{d v} \log F_{j}\left(\xi_{j}\left(s_{i}(v)\right)\right) \geq \frac{d}{d v} \log F_{i}(v)=\frac{d}{d v} \log F_{j}\left(F_{j}^{-1}\left(F_{i}(v)\right)\right)$ in $[z, \bar{v}]$. Finally, 1) and 2) imply that $\log F_{j}\left(\xi_{j}\left(s_{i}(v)\right)\right) \leq \log F_{j}\left(F_{j}^{-1}\left(F_{i}(v)\right)\right)$, which implies that

$$
\xi_{j}\left(s_{i}(v)\right) \leq F_{j}^{-1}\left(F_{i}(v)\right), \forall v \in[z, \bar{v}] .
$$

See, e.g., Milgrom and Weber (1982) and Lebrun (1999). Replacing $v=\xi_{i}(b)$ gives $F_{j}\left(\xi_{j}(b)\right) \leq$ $F_{i}\left(\xi_{i}(b)\right)$. That is, $G_{i}(\cdot) \geq G_{j}(\cdot)$ in $\left[s_{i}(z), \bar{b}\right]$.

\section{Asymmetry in Preferences}

Alternatively, we could assume that bidders are symmetric in private values but asymmetric in preferences, i.e., degrees of risk aversion. Again, we consider the case in which bidders 
have CRRA utility functions. The equilibrium is characterized by

$$
\frac{d}{d b} \xi_{i}(b)=\frac{F\left(\xi_{i}(b)\right)}{(I-1) f\left(\xi_{i}(b)\right)}\left\{\sum_{\imath=1}^{I} \frac{\theta_{\imath}}{\xi_{\imath}(b)-b}-\frac{(I-1) \theta_{i}}{\xi_{i}(b)-b}\right\}
$$

along with the boundary conditions: $\xi_{i}(\bar{b})=\bar{v}$ and $\xi_{i}(\underline{v})=\underline{v}$, where $\xi_{i}(\cdot) \equiv s_{i}^{-1}(\cdot)$ is the inverse bidding strategy of bidder $i . F(\cdot)$ is the common value distribution and $\theta_{i} \in(0,1]$ is bidder $i$ 's CRRA parameter (i.e., $\left.U_{i}(x)=x^{\theta_{i}}\right)$.

Consider two bidders $i$ and $j$ such that $\theta_{i}<\theta_{j}$. Following similar lines, we obtain

$$
\frac{d}{d b} \log F\left(\xi_{j}(b)\right)-\frac{d}{d b} \log F\left(\xi_{i}(b)\right)=\frac{\theta_{i}}{\xi_{i}(b)-b}-\frac{\theta_{j}}{\xi_{j}(b)-b} .
$$

Plugging in $b=s_{i}(v)$ and changing the differentiation to $v$ gives

$$
\frac{d}{d v} \log F\left(\xi_{j}\left(s_{i}(v)\right)\right)=\frac{d}{d v} \log F(v)+s_{i}^{\prime}(v)\left[\frac{\theta_{i}}{v-s_{i}(v)}-\frac{\theta_{j}}{\xi_{j}\left(s_{i}(v)\right)-s_{i}(v)}\right] .
$$

Note that: 1), $\log F(\bar{v})=0$ and $\left.\log F\left(\xi_{j}\left(s_{i}(\bar{v})\right)\right)=0 ; 2\right)$, If $\log F\left(\xi_{j}\left(s_{i}(v)\right)\right)=\log F(v)$, we have $\xi_{j}\left(s_{i}(v)\right)=v$ and then

$$
\frac{\theta_{i}}{v-s_{i}(v)}-\frac{\theta_{j}}{\xi_{j}\left(s_{i}(v)\right)-s_{i}(v)}=\frac{\theta_{i}-\theta_{j}}{v-s_{i}(v)}<0
$$

which implies that $\frac{d}{d v} \log F\left(\xi_{j}\left(s_{i}(v)\right)\right)<\frac{d}{d v} \log F(v)$. In sum, 1) and 2) imply that $\log F\left(\xi_{j}\left(s_{i}(v)\right)\right)>$ $\log F(v)$, which implies that $s_{i}(v)>s_{j}(v), \forall v \in(\underline{v}, \bar{v})$ and $G_{j}(b)>G_{i}(b), \forall b \in(\underline{b}, \bar{b})$. In other words, the more risk averse the bidder is, the higher he/she bids.

\section{References}

Aguirregabiria, V., And P. Mira (2015): "Identification of Games of Incomplete Information with Multiple Equilibria and Common Unobserved Heterogeneity," Working Paper. 
An, Y. (2016): "Identification of First-Price Auctions with Non-Equilibrium Beliefs: A Measurement Error Approach," Working Paper.

An, Y., Y. Hu, And M. Shum (2010): "Estimating first-price auctions with an unknown number of bidders: A misclassification approach," Journal of Econometrics, 157(2), 328341.

Aradillas-López, A., A. Gandhi, And D. Quint (2013): "Identification and inference in ascending auctions with correlated private values," Econometrica, 81(2), 489-534.

Armstrong, T. B. (2013): "Bounds in auctions with unobserved heterogeneity," Quantitative Economics, 4(3), 377-415.

ArYAL, G. (2016): "Identifying Multidimensional Adverse Selection Models," Working Paper, University of Virginia.

Athey, S. (2002): "Monotone comparative statics under uncertainty," Quarterly Journal of Economics, pp. 187-223.

Atkinson, A. B. (1987): "On the measurement of poverty," Econometrica, pp. 749-764.

Baldwin, L. H., R. C. Marshall, and J.-F. Richard (1997): "Bidder collusion at forest service timber sales," Journal of Political Economy, 105(4), 657-699.

BAWA, V. S. (1975): "Optimal rules for ordering uncertain prospects," Journal of Financial Economics, 2(1), 95-121.

BORnKAMP, B., AND K. ICKSTADT (2009): "Bayesian nonparametric estimation of continuous monotone functions with applications to dose-response analysis," Biometrics, 65(1), $198-205$.

Campo, S., E. Guerre, I. Perrigne, and Q. Vuong (2011): "Semiparametric estimation of first-price auctions with risk-averse bidders," The Review of Economic Studies, $78(1), 112-147$. 
Chernozhukov, V., And H. Hong (2004): "Likelihood estimation and inference in a class of nonregular econometric models," Econometrica, 72(5), 1445-1480.

Davidson, R., And J.-Y. Duclos (2000): "Statistical inference for stochastic dominance and for the measurement of poverty and inequality," Econometrica, 68(6), 1435-1464. (2013): "Testing for restricted stochastic dominance," Econometric Reviews, 32(1), $84-125$.

Diaconis, P., AND D. Ylvisaker (1985): "Quantifying prior opinion," Bayesian statistics, $2,133-156$.

Donald, S. G., And H. J. PaARsch (1993): "Piecewise pseudo-maximum likelihood estimation in empirical models of auctions," International Economic Review, pp. 121-148.

FANG, H., AND X. TANG (2014): "Inference of bidders' risk attitudes in ascending auctions with endogenous entry," Journal of Econometrics, 180(2), 198-216.

Flambard, V., And I. Perrigne (2006): "Asymmetry in procurement auctions: evidence from snow removal contracts," The Economic Journal, 116(514), 1014-1036.

Fox, J. T., AND A. GANDhi (2016): "Nonparametric identification and estimation of random coefficients in multinomial choice models," The RAND Journal of Economics, 47(1), 118-139.

Fox, J. T., K. IL Kim, S. P. Ryan, and P. Bajari (2012): "The random coefficients logit model is identified," Journal of Econometrics, 166(2), 204-212.

Gentry, M., And T. Li (2014): "Identification in auctions with selective entry," Econometrica, 82(1), 315-344.

Gimenes, N., And E. Guerre (2016): "Quantile Regression Methods for First-Price Auction: A Signal Approach," Working Paper. 
GrundL, S., AND Y. ZHU (2015): "Identification and Estimation of Risk Aversion in First Price Auctions with Unobserved Auction Heterogeneity," Working Paper.

Guerre, E., I. Perrigne, and Q. Vuong (2000): "Optimal Nonparametric Estimation of First-price Auctions," Econometrica, 68(3), 525-574.

(2009): "Nonparametric Identification of Risk Aversion in First-Price Auctions Under Exclusion Restrictions," Econometrica, 77(4), 1193-1227.

HaILe, P. A. (2001): "Auctions with resale markets: an application to US forest service timber sales," American Economic Review, pp. 399-427.

Haile, P. A., H. Hong, And M. Shum (2003): "Nonparametric tests for common values at first-price sealed-bid auctions," Discussion paper, National Bureau of Economic Research.

Haile, P. A., And E. Tamer (2003): "Inference with an incomplete model of English auctions," Journal of Political Economy, 111(1), 1-51.

Hirano, K., and J. R. Porter (2003): "Asymptotic Efficiency in Parametric Structural Models with Parameter-Dependent Support," Econometrica, 71(5), 1307-1338.

Hu, Y. (2008): "Identification and estimation of nonlinear models with misclassification error using instrumental variables: A general solution," Journal of Econometrics, 144(1), $27-61$.

L (2015): "Microeconomic models with latent variables: applications of measurement error models in empirical industrial organization and labor economics," Working Paper.

Hu, Y., D. McAdams, and M. Shum (2013): "Identification of first-price auctions with non-separable unobserved heterogeneity," Journal of Econometrics, 174(2), 186-193.

Hu, Y., and S. M. Schennach (2008): "Instrumental variable treatment of nonclassical measurement error models," Econometrica, 76(1), 195-216. 
KIM, D.-H. (2015): "Nonparametric estimation of utility function in first-price sealed-bid auctions," Economics Letters, 126, 101-106.

KRASNOKUTSKAYA, E. (2011): "Identification and estimation of auction models with unobserved heterogeneity," The Review of Economic Studies, 78(1), 293-327.

(2012): "Identification And Estimation Of Auction Model With Two-dimensional Unobserved Heterogeneity," International Economic Review, 53(3), 659-692.

Krishna, V. (2009): Auction theory. Academic press.

LAMY, L. (2012): "The econometrics of auctions with asymmetric anonymous bidders," Journal of Econometrics, 167(1), 113-132.

LeBrun, B. (1999): "First price auctions in the asymmetric $\mathrm{N}$ bidder case," International Economic Review, pp. 125-142.

Li, T., I. Perrigne, And Q. Vuong (2000): "Conditionally independent private information in OCS wildcat auctions," Journal of Econometrics, 98(1), 129-161.

Li, T., AND Q. VuONG (1998): "Nonparametric estimation of the measurement error model using multiple indicators," Journal of Multivariate Analysis, 65(2), 139-165.

LiU, N., AND Y. LuO (2016): "A Nonparametric Test for Comparing Valuation Distributions in First-Price Auctions," Working Paper, University of Toronto.

Lu, J., AND I. PERrigne (2008): "Estimating risk aversion from ascending and sealedbid auctions: the case of timber auction data," Journal of Applied Econometrics, 23(7), $871-896$.

Maskin, E., And J. Riley (2000): "Asymmetric auctions," Review of Economic studies, pp. $413-438$. 
Milgrom, P. R., And R. J. Weber (1982): "A theory of auctions and competitive bidding," Econometrica, pp. 1089-1122.

Quint, D. (2015): "Identification in Symmetric English Auctions with Additively Separable Unobserved Heterogeneity," Working Paper.

Roberts, J. W. (2013): "Unobserved heterogeneity and reserve prices in auctions," The RAND Journal of Economics, 44(4), 712-732.

VAn Dorp, J. R., And S. Kotz (2002): "The standard two-sided power distribution and its properties: with applications in financial engineering," The American Statistician, 56(2), 90-99.

XIAO, R. (2015): "Identification and Estimation of Incomplete Information Games with Multiple Equilibria," Available at SSRN.

— (2016): "Nonparametric Identification of Dynamic Games with Multiple Equilibria and Unobserved Heterogeneity," Working Paper.

Zincenko, F. (2014): "Nonparametric Estimation of First-Price Auctions with Risk-Averse Bidders," Working Paper. 